\title{
Article \\ Graft Compatibility Classification within Aurantioideae Based on Biometric Traits and the Anatomy of Graft Union
}

\author{
Laudecir Lemos Raiol-Junior 1,2 (D), Everton Vieira de Carvalho ${ }^{1,2,+}$, Alécio Souza Moreira ${ }^{1,2,+}+^{(D)}$, \\ João Paulo Rodrigues Marques ${ }^{3,+}+\mathbb{D}$, Eduardo Sanches Stuchi ${ }^{1,4}$, Leandro Peña ${ }^{2,5}$ \\ and Eduardo Augusto Girardi 1,2,*(D)
}

check for

updates

Citation: Raiol-Junior, L.L.; de Carvalho, E.V.; Moreira, A.S.; Marques, J.P.R.; Stuchi, E.S.; Peña, L.; Girardi, E.A. Graft Compatibility Classification within Aurantioideae Based on Biometric Traits and the Anatomy of Graft Union. Agriculture 2022, 12, 76. https://doi.org/ 10.3390/agriculture12010076

Academic Editor: Yuan Huang

Received: 10 November 2021

Accepted: 29 December 2021

Published: 6 January 2022

Publisher's Note: MDPI stays neutral with regard to jurisdictional claims in published maps and institutional affiliations.

Copyright: () 2022 by the authors. Licensee MDPI, Basel, Switzerland. This article is an open access article distributed under the terms and conditions of the Creative Commons Attribution (CC BY) license (https:// creativecommons.org/licenses/by/ $4.0 /)$.
1 Embrapa Cassava \& Fruits, Cruz das Almas 44380-000, BA, Brazil; laudecir.raiol@unesp.br (L.L.R.-J.); everton.carvalho@unesp.br (E.V.d.C.); alecio.moreira@embrapa.br (A.S.M.); eduardo.stuchi@embrapa.br (E.S.S.)

2 Fund for Citrus Protection, Araraquara 14807-040, SP, Brazil; lpenya@ibmcp.upv.es

3 Department of Basic Science, Faculty of Animal Science and Food Engineering, University of São Paulo, Pirassununga 13635-900, SP, Brazil; jprmarques@usp.br

4 Coopercitrus Credicitrus Foundation, Bebedouro 14713-000, SP, Brazil

5 Institute of Molecular and Cellular Plant Biology, Higher Council for Scientific Research, Polytechnic University of Valencia, 46022 Valencia, Spain

* Correspondence: eduardo.girardi@embrapa.br

+ These authors contributed equally to this work.

\begin{abstract}
Citrus relatives are a relevant source of valuable traits for use in citrus breeding, including resistance to diseases such as Huanglongbing (HLB). Resistant rootstocks may impact tree responses to HLB. This requires graft compatibility, which has been poorly investigated within the Aurantioideae. In this study, the biometric characteristics and the anatomy of the graft union of 86 scion/rootstock combinations were assessed. This comprised 18 genotypes/species and 8 genera from Citrinae, Balsamocitrinae, and Clauseninae subtribes sensu Swingle and Reece. Most graft combinations were found to be noncompatible. Phylogenetic proximity did not ensure successful grafting as, for example, Orange jasmine autografts failed, whereas some intergeneric grafts were successful ( $>60 \%$ of graft-take). Plant scion height was directly related to graft-take, but the correlation between the scion and rootstock stem diameters was not a reliable indicator of graft compatibility. Rangpur/Tabog, Tabog/Rangpur, Wampee/Rangpur, Wampee/Pomeroy, Wampee/Swingle, Pomeroy/Wampee, and Swingle/Wampee were the most compatible intergeneric graft combinations. Graft-take success for this was at similar levels to those of sweet orange grafted on common citrus rootstocks. The position as a scion or rootstock in the combination affected the performance and was specific to the genotypes tested. The lack of differentiation between xylem-derived calli and the accumulation of phenolic compounds at the graft union were clear anatomical and biochemical markers, respectively, of incompatibility for most Aurantioideae combinations. In the field, within a set of the ten most promising combinations, Hamlin/Rangpur (control) was the only one that became infected by 'Candidatus Liberibacter asiaticus'. This was first observed 12 months after planting. Overall, the assessment of biometric traits and anatomy of the graft union allowed Aurantioideae genotypes to be divided into four clusters, with respect to their graft compatibility, as follows: fully compatible with high graft-take and plant growth; potentially compatible with high graft-take but lower plant growth; partially incompatible with lower graft-take and poor plant growth; and fully incompatible with a complete absence of graft-take.
\end{abstract}

Keywords: Citrus spp.; Citreae; Clausenae; Huanglongbing; citrus relatives; rootstock

\section{Introduction}

Aurantioideae is one of the largest Rutaceae subfamilies [1-3]. It is divided into the Clauseneae and Citreae tribes. These are composed of 33 genera and 210 species, according 
to Swingle and Reece [1]. The Clauseneae tribe encompasses the most distant citrus relatives, which typically have pinnate leaves and small fruits. Citreae contains "citrus fruit trees" in the Citrinae subtribe. This includes the genus Citrus, which is currently known to have a few true species and thousands of intrageneric complex citrus hybrids and types with relevant commercial importance [4-6]. The broad genetic and phenotypic diversity observed within Aurantioideae may be an important source of traits that could be used to improve significant attributes in commercial citrus. This includes yield, abiotic stress tolerance, and pest and disease resistance [6-9].

Citrus trees are commercially propagated by grafting, in which the canopy is the scion variety and the underground part is the rootstock. Each of these may have specific traits of interest. There is an interest in crossbreeding between Citrus and closely related genera, such as Fortunella, Microcitrus, Eremocitrus, and Poncirus [1,10], to obtain new varieties because they are sexually compatible. However, most citrus relatives are sexually incompatible with Citrus [11,12]. An alternative to take advantage of the Aurantioideae gene pool is their direct use as a rootstock for commercial citrus types/species. Grafting may, therefore, be used to assess traits of interest within a wide range of citrus relatives. A few studies have evaluated the performance and graft compatibility in citrus scions and citrus relatives. This has produced varied results, depending on the combinations and experimental conditions used [13-18].

Intergeneric graft compatibility results from a complex sequential process that still needs to be better understood. For successful grafting, the initial injury response should be followed by a rapid healing process with the accumulation of phenolic and lipophilic compounds. A proliferation of parenchymal cells then occurs to form undifferentiated callus tissue. This is followed by the differentiation of the cambial cells to establish junctions with vascular vessels, allowing connection between the scion and rootstock genotypes [19]. However, graft incompatibility may arise from the irregular alignment of vascular exchange tissues [20,21], unknown genetic or cellular factors [22,23], production and accumulation of certain detrimental phenolic compounds [24-26], phytohormone imbalance [27,28], and ion disturbances $[29,30]$. Phylogenetically distant genotypes are more likely to exhibit graft incompatibility [31,32]. Therefore, the taxonomic affinity between the two target species is the first step to be considered prior to proceeding with this technique. Graft compatibility of intergeneric and intrafamilial species represents huge agronomic potential for genetic improvement and better crop management through the use of interesting characteristics from wild relative rootstocks in combination with commercial citrus scion varieties.

More recently, there has been increased interest in using genera from Aurantioideae as a source of resistance to the bacterium 'Candidatus Liberibacter asiaticus' (CLas), which is associated with Huanglongbing (HLB) $[7,8,33,34]$. This is one of the most destructive diseases affecting citrus trees worldwide [35]. In the HLB pathosystem, the rootstock plays an important role in the progression of disease symptoms, with the loss of root biomass reported as being one of the first noticeable symptoms of the disease [36]. Rootstocks that are considered less susceptible, such as Poncirus trifoliata and its hybrids, have shown different levels of disease severity. This has varied from minimal symptoms in ungrafted seedling plants to extensive damage in graft combinations with other scion varieties. These disease responses are also likely influenced by the environmental and management conditions [37-41]. It is, therefore, necessary to evaluate other genera with resistance against CLas infection. Intergeneric grafting has the potential to combine truly HLB-resistant rootstocks with susceptible citrus scions. This technique is widely used in fruit trees for disease management with horticulturally viable graft combinations [31,32]. There are multiple successful examples of growers overcoming citrus diseases by grafting susceptible scion varieties onto disease-resistant/tolerant rootstocks. This strategy has been used to combat citrus diseases such as Citrus tristeza virus infections [14,42,43], citrus variegated chlorosis caused by Xylella fastidiosa [44,45], foot rot caused by Phytophthora spp., and citrus blight $[46,47]$. This is also a strategy that can be used to control diseases in crops such as apples, grapevines, and some vegetables [48-52]. 
For the current study, a total of 86 scion/rootstock (S/R) combinations were screened. These comprised 18 species from eight genera of the Aurantioideae subfamily. They were screened for graft compatibility, based on biometric traits and anatomical evaluation of graft union for up to 21 months after grafting (MAG) in greenhouse. In addition, the initial horticultural performance and reactions to HLB were assessed among the most suitable combinations under field conditions.

\section{Materials and Methods}

\subsection{Plant Material}

Taxonomic classification of Aurantioideae plants was performed as proposed by Swingle and Reece [1] with modifications reported by Ollitrault et al. [53] (Table 1). Graft compatibility was evaluated between commercial varieties of Citrus types and eight genera from Aurantioideae. Seedlings were cultivated in cone-shaped $50 \mathrm{~mL}$ plastic tubes that were filled with coir. Nucellar plants were visually selected within polyembryonic species. The species used in this study were selected based on a review of literature describing responses to CLas, the bacterium associated with HLB pathogenesis. Responses to the bacterium ranged from complete immunity $[8,34]$ to partial resistance or transient/temporary multiplication of the pathogen [33], that is, initial multiplication gradually decreased until the pathogen became absent following inoculation. In other cases, there was a very low bacterial multiplication within plant tissues [54,55] or varying levels of susceptibility to the disease $[7,8,39,41,56]$. All seedlings were grown in screenhouses in Araraquara, Sao Paulo State, Brazil.

Table 1. Phylogenetic classification (PC) of the Aurantioideae species used in this work.

\begin{tabular}{|c|c|c|c|c|c|}
\hline Species ${ }^{1}$ & Genotype Name & Tribe/Subtribe ${ }^{2}$ & Type & PC & $\begin{array}{l}\text { CLas Infection } \\
\text { Classification- } \\
\text { Authors }^{3}\end{array}$ \\
\hline Citrus. $\times$ aurantium var. sinensis L. & Hamlin & Citreae/Citrinae & True citrus & 1 & HS [41] \\
\hline C. $\times$ aurantium var. sinensis $\mathrm{L}$. & Pera & Citreae/Citrinae & True citrus & 1 & HS [7] \\
\hline C. $\times$ aurantium var. sinensis $\mathrm{L}$. & Pineapple & Citreae/Citrinae & True citrus & 1 & HS [8] \\
\hline C. $\times$ aurantium var. sinensis $\mathrm{L}$. & Rubi & Citreae/Citrinae & True citrus & 1 & HS [39] \\
\hline C. $\times$ aurantium var. sinensis $\mathrm{L}$. & Valencia & Citreae/Citrinae & True citrus & 1 & HS [41] \\
\hline C. $\times$ aurantium L. var. aurantium & Sour orange & Citreae/Citrinae & True citrus & 1 & HS [8] \\
\hline C. $\times$ limon var. limon (L.) Burm. f. & Lemon & Citreae/Citrinae & True citrus & 1 & HS [8] \\
\hline C. $\times$ limonia Osbeck var. limonia & Rangpur & Citreae/Citrinae & True citrus & 1 & HS [8] \\
\hline C. reticulata Blanco & Ponkan & Citreae/Citrinae & True citrus & 1 & HS [8] \\
\hline C. paradisi Macfad. $\times$ P. trifoliata & Swingle & Citreae/Citrinae & Trifoliate & 2 & MS [41] \\
\hline Poncirus trifoliata (L.) Raf. & Pomeroy & Citreae/Citrinae & Trifoliate & 2 & MS [7] \\
\hline $\begin{array}{l}\text { Atalantia buxifolia (Poir.) Oliv. (syn. } \\
\text { Severinia buxifolia) }\end{array}$ & Box orange & Citreae/Citrinae & Small-fruit citrus & 3 & HS [56] \\
\hline Swinglea glutinosa (Blanco) Merr. & Tabog & $\begin{array}{l}\text { Citreae/ } \\
\text { Balsamocitrinae }\end{array}$ & Hard-shell & 4 & $\mathrm{TM}[33]$ \\
\hline Aegle marmelos (L.) Corr. & Bael & $\begin{array}{l}\text { Citreae/ } \\
\text { Balsamocitrinae }\end{array}$ & Hard-shell & 4 & $\mathrm{~T}[8]$ \\
\hline Murraya koenigii (L.) Spreng & Curry tree & $\begin{array}{l}\text { Clausenae/ } \\
\text { Clauseninae }\end{array}$ & Remote citrus & 5 & I [34] \\
\hline M. paniculata (L.) Jack & Orange jasmine & $\begin{array}{l}\text { Clausenae/ } \\
\text { Clauseninae }\end{array}$ & Remote citrus & 5 & TM [33] \\
\hline Clausena lansium (Lour.) Skeels & Wampee & $\begin{array}{l}\text { Clausenae/ } \\
\text { Clauseninae }\end{array}$ & Remote citrus & 5 & LT $[54,55]$ \\
\hline Glycosmis pentaphylla (Retz.) Correa & Orange berry & $\begin{array}{l}\text { Clausenae/ } \\
\text { Clauseninae }\end{array}$ & Remote citrus & 5 & $\mathrm{I}[8]$ \\
\hline
\end{tabular}

${ }^{1}$ Scientific nomenclature according to USDA. Available online: https://npgsweb.ars-grin.gov/gringlobal/taxon/ taxonomysearch?t=pnlspecies (accessed on 27 September 2021). ${ }^{2}$ Taxonomic classifications according to Swingle and Reece [1]. ${ }^{3}$ Classification of the species regarding susceptibility to 'Candidatus Liberibacter asiaticus' (CLas) infection: HS, highly susceptible; MS, moderately susceptible; TM, transient or temporary multiplication; LT, low bacterial titer; T, tolerant; I, immune. 


\subsection{Grafting Procedures}

Six months after seeds were sown, the seedlings had grown to a height of $35 \pm 10 \mathrm{~cm}$ and had reached a stem diameter of $4 \pm 2 \mathrm{~mm}$. They were then grafted using top cleft grafting. All genotypes were evaluated for use as both the scion and the rootstock. Grafting was reciprocal, forming different combinations to examine the effect of the genotype position on the grafted plant. Combinations with "Hamlin" and "Valencia" sweet oranges grafted onto "Rangpur" lime were used as controls. These combinations were selected because of their extensive commercial use with the production of successful grafts. For each graft combination, one to seventy plants were grafted and used as replicates. However, only those with a minimum of three replicates were statistically analyzed.

The rootstock was topped with scissors at $10 \pm 5 \mathrm{~cm}$ height. The stem was then split in half at a depth of $0.5 \mathrm{~cm}$ using a pocketknife. The scion graft consisted of $5 \mathrm{~cm}$ long segments that were collected from seedlings with a stem diameter similar to that of the rootstock. The scion was made by cutting a long and gradually tapering wedge to maintain an equivalent length to the depth of the slit made in the rootstock stem. The scion was inserted carefully into the rootstock to facilitate cambium layer matching. After the scion was placed, the entire union was thoroughly wrapped with plastic strips. A humid chamber was used, enveloping the scion region with a $10 \times 20 \mathrm{~cm}$ plastic bag. This was fixed to the rootstock base with wire and kept for 30 days. The plants were maintained in a climate-controlled greenhouse at an average temperature of $26 \pm 2{ }^{\circ} \mathrm{C}$. The plants were kept in cone-shaped plastic tubes with periodic irrigation for approximately 60 days with a nutrient solution composed of the following (g/1000 L): calcium nitrate (467), magnesium sulfate (679), potassium nitrate (72), monoammonium phosphate (18.8), phosphoric acid (22), Fe-EDHHA (35), Cu-EDTA (18.7), Zn-EDTA (4), Mn-EDTA (3.1), boric acid (2), and sodium molybdate (0.3).

Two groups of grafted plants were used and evaluated as part of separate experiments. The first group comprised 56 scion and rootstock combinations (Experiment 1). The plants were evaluated in the greenhouse for 21 months (May 2018 to February 2020). The combinations with the most potential were then planted in the field to evaluate their longterm graft compatibility. Their initial agronomic performance and reaction to CLas infection was also examined. The second group comprised 39 scion and rootstock combinations (Experiment 2). A total of 12 grafted plants from this group were monitored to validate the combinations with the most potential (i.e., the ones from the Experiment 1), with 27 new combinations also being tested. In Experiment 2, plants were grafted and evaluated for up to 13-16 months in the greenhouse. This included three different sets of plants cultivated from August 2019 to July 2021, which were grouped for performing statistical analysis.

\subsection{Graft-Take and Plant Growth}

All the plants were observed for 2 MAG to evaluate graft compatibility at the time of transplanting onto larger recipients in the greenhouse. Plants in which the grafting union had healed, and the presence of new shoots was observed were considered to have initial compatibility. The plants were transplanted into $30 \mathrm{~cm} \times 20 \mathrm{~cm}$ black polyethylene bags that were filled with decomposed pine bark substrate (Terra do Paraíso, Holambra, São Paulo, Brazil). They were then transferred to a greenhouse. Plants were fertigated every three days with the same nutrient solution as described previously in Section 2.2. Plants in the first group (Experiment 1) were further evaluated at 7, 14, and 21 months after cleft grafting, while plants in the second group (Experiment 2) were evaluated from 7 to 12 months and from 13 to 16 months after cleft grafting. The number of grafts alive was counted for each treatment, with the time after grafting also being recorded. The percentage relative to the total number of grafted plants was calculated as the graft-take. On all assessment dates, the scion and rootstock stem diameters were measured $5 \mathrm{~cm}$ above and below the graft union [57] with a digital caliper. The correlations between the scion and rootstock stem diameters were then calculated. Plant height was measured from the collar end to the apex of the plant using a ruler. Only data from the final date when plants 
were still alive were presented, thereby encompassing the maximum plant growth attained by each evaluated S/R combination.

\subsection{Anatomical Evaluations}

Twenty-four S/R combinations from the second grafting group were selected for anatomical assessment between 14 and 26 MAG. Three plants from each S/R combination were longitudinally sectioned with a scalpel blade at the median region of the grafted region in a $5 \mathrm{~cm}$ long stem segment, splitting them into two halves used for sampling. One sample was then stained a pink color with phloroglucin $(0.1 \%)$ for $5 \mathrm{~min}$ to detect the lignified cell walls. The other was stained a brown color with $5 \%$ ferric chloride for $2 \mathrm{~h}$ to detect phenolic compounds [58]. All samples were then analyzed using a digital microscope (KH-8700, Hirox, NJ, USA), with the images being digitally registered.

\subsection{Field Cultivation and CLas Detection}

In June 2020, 21-month-old plants from the 10 graft combinations with the most potential were planted in the field in the municipality of Bebedouro, São Paulo State, Brazil $\left(20^{\circ} 53^{\prime} 16^{\prime \prime} \mathrm{S}, 48^{\circ} 28^{\prime} 11^{\prime \prime} \mathrm{W}, 600 \mathrm{~m}\right.$ a.s.l.) for Experiment 3. Using this approach, the main objective was to evaluate the initial horticultural performance of the selected graft combinations, as well as disease progression after natural inoculation, under field conditions. The local climate was classified as Cwa. This was characterized as mountain subtropical (Köppen-Geiger climate classification system) [59]. The soil type in the experimental area was classified as Red dystrophic oxisol. Horticultural practices followed standard recommendations for citrus cultivation in Brazil. The insect vector of CLas (Diaphorina citri Kuwayama) was controlled using monthly spraying on a rotation system using contact insecticides. This was to prevent the uncontrolled increase and movement of the vector population to the surrounding areas. Systemic insecticides were not used in the drenches. Twelve months after planting, plant survival was calculated as the relationship between the number of trees alive and the total number of planted trees. The scion and rootstock stem diameters and plant height were measured as described in Section 2.3. In addition, the presence of typical symptoms of HLB was visually evaluated. If CLas was present, five symptomatic leaves were collected. In the absence of symptoms, five leaves from the last shoot flush were collected and assessed for CLas presence. This was done via quantitative or real-time polymerase chain reaction (qPCR) in each plant from all S/R combinations. Leaves were washed with water and soap, and $0.3 \mathrm{~g}$ of tissue from the midrib was used for extraction of total genomic DNA using cetyltrimethylammonium bromide (CTAB) [60], as described by Teixeira et al. [61]. qPCR was performed for detection of the 16s rRNA from CLas as adapted from [62]; the reaction mixture comprised $1 \mu \mathrm{L}$ of total DNA $(100 \mathrm{ng} / \mu \mathrm{L})$, TaqMan R PCR master mix $(1 \times)$ (Invitrogen, Carlsbad, CA, USA), and RNRf/RNRr primers/probe $(0.3 \mu \mathrm{M} / 0.05 \mu \mathrm{M})$, in a total volume of $12 \mu \mathrm{L}$. qPCR was performed using a thermocycler (StepOnePlus, Applied Biosystems, Foster City, CA, USA). Samples were considered CLas-positive when the cycle threshold $(\mathrm{Ct})$ value was equal to or lower than 35.0.

\subsection{Data Analyses}

All graft-take data from the S/R combinations were treated as binary data (" 1 " for successful and " 0 " for unsuccessful graft-take). The binary data were analyzed via a binomial general linear model using the $\mathrm{glm}$ package [63]. For significant differences $(p<0.05)$, mean values were separated using the Scott-Knott (SK) clustering analysis [64]. Height, scion diameter, rootstock diameter, and scion/rootstock diameter relation, when complied with the assumption of normal distribution of the residuals, were subjected to analysis of variance using the $\mathrm{F}$ test. For significant differences $(p<0.05)$, mean values were grouped using the SK clustering analysis [65]. When normality assumptions for the residual distributions were not met, the data were analyzed using the Kruskal-Wallis test. 
Mean values composed of less than three biological replicates for any variable/treatment were not statistically analyzed.

For hierarchical grouping of the evaluated S/R combinations and principal component analysis (PCA), the data were standardized. The Euclidean distance was used to measure similarity, and Ward's method was applied to assess linkage [66]. Cluster analysis was performed using the $\mathrm{NbClust}$ package in R 3.6.1 [67]. PCA was performed using the FactoMineR package in R 3.6.1 [68]. Only components with eigenvalues $>1$ were considered [69]. Maximum plant height, scion and rootstock stem diameters, scion/rootstock diameter relation, and graft-take percentage from the last assessment were used in the PCA. Pearson correlation analyses were performed to assess the relationships between the variables. All analyses were performed using the R statistical software, version 3.6.1 [63].

\section{Results}

\subsection{First Group of S/R Combinations (Experiment 1)}

The S/R combinations analyzed in the first group presented $0-100 \%$ graft-take in all assessments. There were 11 clusters in the first three assessments and eight in the last assessment, $21 \mathrm{MAG}$, according to the SK test. Ten S/R combinations (17.9\%) showed more than $60 \%$ graft-take, with the average plant height being higher than $22 \mathrm{~cm}$ (Table 2). In addition, the average scion stem diameter (SD) for these combinations ranged from 2.64 to $8.36 \mathrm{~mm}$ for the first five SK clusters. The average rootstock stem diameter (RD) ranged from 3.46 to $9.26 \mathrm{~mm}$ for the first six SK clusters. The average SD/RD relation ranged from 0.52 to 1.71 for the first four SK clusters (Table 2). The best-performing S/R combinations belonged to distinct phylogenetic groups (PGs) 1,3, 4, and 5 (Table 1). In contrast, 43 of the $56 \mathrm{~S} / \mathrm{R}$ combinations $(76.8 \%$ ) showed less than 10\% graft-take at 21 MAG (Table 2).

Table 2. Graft-take percentage of the first group of scion/rootstock genotypes combinations within different Aurantioideae phylogenetic groups (PGs) at 2, 7, 14, and 21 months after grafting (MAG), mean values of plant height $(\mathrm{H})$, scion stem diameter $(\mathrm{SD})$, rootstock stem diameter $(\mathrm{RD})$, scion and rootstock stem diameter relation (SD/RD) at 14 MAG or before plant's death, and graft compatibility group (GC).

\begin{tabular}{|c|c|c|c|c|c|c|c|c|c|c|c|c|}
\hline \multirow{2}{*}{ Scion } & \multirow{2}{*}{ Rootstock } & \multirow{2}{*}{$\mathrm{PG}^{1}$} & \multirow{2}{*}{$\mathbf{N}^{*}$} & \multicolumn{4}{|c|}{ MAG } & \multirow{2}{*}{$\begin{array}{c}\mathrm{H} \\
(\mathrm{cm})\end{array}$} & \multirow{2}{*}{$\begin{array}{c}\text { SD } \\
(\mathrm{mm})\end{array}$} & \multirow{2}{*}{$\begin{array}{c}\text { RD } \\
(\mathrm{mm})\end{array}$} & \multirow{2}{*}{ SD/RD } & \multirow{2}{*}{$\mathrm{GC}^{3}$} \\
\hline & & & & 2 & 7 & 14 & $21^{2}$ & & & & & \\
\hline Hamlin & Rangpur & $1 / 1$ & $10-20$ & $100.00 \mathrm{a}$ & $100.00 \mathrm{a}$ & $100.00 \mathrm{a}$ & $100.00 \mathrm{a}$ & $74.20 \mathrm{a}$ & $6.68 b$ & $8.94 a$ & $0.76 \mathrm{c}$ & 1 \\
\hline Rangpur & Tabog & $1 / 4$ & 25 & $100.00 \mathrm{a}$ & $100.00 \mathrm{a}$ & $100.00 \mathrm{a}$ & $100.00 \mathrm{a}$ & $61.88 \mathrm{a}$ & $6.98 b$ & $6.08 c$ & $1.18 \mathrm{~b}$ & 1 \\
\hline Rangpur & Hamlin & $1 / 1$ & 20 & $100.00 \mathrm{a}$ & $100.00 \mathrm{a}$ & $95.00 \mathrm{~b}$ & $95.00 \mathrm{~b}$ & $82.89 a$ & $7.01 \mathrm{~b}$ & $9.26 a$ & $0.76 c$ & 1 \\
\hline Wampee & Rangpur & $5 / 1$ & 10 & $100.00 \mathrm{a}$ & $90.00 c$ & $90.00 c$ & $90.00 c$ & $36.22 c$ & $8.36 a$ & $4.95 \mathrm{~d}$ & $1.71 \mathrm{a}$ & 1 \\
\hline Rangpur & Wampee & $1 / 5$ & 10 & $100.00 \mathrm{a}$ & $100.00 \mathrm{a}$ & $100.00 \mathrm{a}$ & $90.00 \mathrm{c}$ & $22.20 \mathrm{~d}$ & $4.37 d$ & $3.46 \mathrm{f}$ & $1.27 \mathrm{~b}$ & 1 \\
\hline Box orange & Tabog & $3 / 4$ & 17 & $100.00 \mathrm{a}$ & $100.00 \mathrm{a}$ & $94.12 b$ & $88.24 c$ & $50.31 b$ & $4.63 \mathrm{~d}$ & $5.31 d$ & $0.88 c$ & 1 \\
\hline Tabog & Box orange & $4 / 3$ & 17 & $100.00 \mathrm{a}$ & $100.00 \mathrm{a}$ & $94.12 b$ & $88.24 c$ & $37.44 \mathrm{c}$ & $4.05 \mathrm{~d}$ & $5.47 \mathrm{~d}$ & $0.75 c$ & 1 \\
\hline Tabog & Rangpur & $4 / 1$ & 25 & $100.00 \mathrm{a}$ & $100.00 \mathrm{a}$ & $100.00 \mathrm{a}$ & $88.00 \mathrm{c}$ & $71.28 \mathrm{a}$ & $5.50 c$ & $7.63 b$ & $0.72 \mathrm{c}$ & 1 \\
\hline Hamlin & Box orange & $1 / 3$ & $10-15$ & $100.00 \mathrm{a}$ & $100.00 \mathrm{a}$ & $100.00 \mathrm{a}$ & $73.33 d$ & $32.50 \mathrm{c}$ & $3.40 \mathrm{e}$ & $5.89 c$ & $0.58 \mathrm{~d}$ & 1 \\
\hline Box orange & Hamlin & $3 / 1$ & $10-15$ & $100.00 \mathrm{a}$ & $100.00 \mathrm{a}$ & $100.00 \mathrm{a}$ & $60.00 \mathrm{e}$ & $22.60 d$ & $2.64 \mathrm{e}$ & $5.05 \mathrm{~d}$ & $0.52 \mathrm{~d}$ & 1 \\
\hline Rangpur & Bael & $1 / 4$ & 8 & $87.50 c$ & $87.50 \mathrm{c}$ & $87.50 c$ & $50.00 \mathrm{e}$ & $50.14 b$ & $4.46 \mathrm{~d}$ & $7.51 b$ & $0.63 d$ & 1 \\
\hline Tabog & Wampee & $4 / 5$ & 5 & $100.00 \mathrm{a}$ & $100.00 \mathrm{a}$ & $80.00 \mathrm{~d}$ & $20.00 \mathrm{f}$ & $18.75 d$ & $3.65 d$ & $3.87 \mathrm{f}$ & $0.94 b$ & 2 \\
\hline Orange jasmine & Orange jasmine & $5 / 5$ & 38 & $36.84 f$ & $18.42 \mathrm{~g}$ & $18.42 \mathrm{~g}$ & $15.79 \mathrm{f}$ & $64.29 \mathrm{a}$ & $5.26 \mathrm{c}$ & $6.39 c$ & $0.82 \mathrm{c}$ & 1 \\
\hline Orange jasmine & Pomeroy & $5 / 2$ & 45 & $80.00 \mathrm{~d}$ & $62.22 \mathrm{~d}$ & $35.56 f$ & $6.67 \mathrm{~g}$ & $20.06 \mathrm{~d}$ & $3.52 \mathrm{e}$ & $4.57 \mathrm{e}$ & $0.79 c$ & 2 \\
\hline Curry tree & Rangpur & $5 / 1$ & 20 & $95.00 \mathrm{~b}$ & $85.00 \mathrm{c}$ & $35.00 \mathrm{f}$ & $5.00 \mathrm{~g}$ & $24.00 \mathrm{~d}$ & $3.20 \mathrm{e}$ & $5.38 \mathrm{~d}$ & $0.62 \mathrm{~d}$ & 2 \\
\hline Rangpur & Curry tree & $1 / 5$ & 20 & $70.00 \mathrm{e}$ & $55.00 \mathrm{~d}$ & $10.00 \mathrm{~h}$ & $5.00 \mathrm{~g}$ & $23.00^{4}$ & $6.08^{4}$ & $3.71^{4}$ & $1.64^{4}$ & 2 \\
\hline Rubi & Orange jasmine & $1 / 5$ & 15 & $60.00 \mathrm{e}$ & $6.67 i$ & $6.67 i$ & $0.00 \mathrm{~h}$ & $40.00^{4}$ & $5.75^{4}$ & $9.12^{4}$ & $0.63^{4}$ & 1 \\
\hline Sicilian lemon & Orange jasmine & $1 / 5$ & 30 & $3.33 \mathrm{j}$ & $3.33 \mathrm{j}$ & $0.00 \mathrm{k}$ & $0.00 \mathrm{~h}$ & $36.00^{4,5}$ & $4.40^{4,5}$ & $5.00^{4,5}$ & $0.88^{4,5}$ & 2 \\
\hline Tabog & Orange jasmine & $4 / 5$ & 30 & $33.33 \mathrm{f}$ & $13.33 \mathrm{~h}$ & $3.33 \mathrm{j}$ & $0.00 \mathrm{~h}$ & $27.00^{4}$ & $4.32^{4}$ & $5.80^{4}$ & $0.74^{4}$ & 2 \\
\hline Orange berry & Rangpur & $5 / 1$ & 9 & $88.89 \mathrm{c}$ & $55.56 \mathrm{~d}$ & $22.22 \mathrm{~g}$ & $0.00 \mathrm{~h}$ & $21.00^{4}$ & $3.21^{4}$ & $3.96^{4}$ & $0.83^{4}$ & 2 \\
\hline Bael & Rangpur & $4 / 1$ & 8 & $75.00 \mathrm{~d}$ & $50.00 \mathrm{~d}$ & $12.50 \mathrm{~h}$ & $0.00 \mathrm{~h}$ & $21.00^{4}$ & $2.37^{4}$ & $3.21^{4}$ & $0.74^{4}$ & 2 \\
\hline Orange jasmine & Tabog & $5 / 4$ & 30 & $40.00 \mathrm{f}$ & $26.67 f$ & $0.00 \mathrm{k}$ & $0.00 \mathrm{~h}$ & $20.64^{5}$ & $3.44^{5}$ & $4.30^{5}$ & $0.80^{5}$ & 2 \\
\hline Wampee & Curry tree & $5 / 5$ & 5 & $100.00 \mathrm{a}$ & $100.00 \mathrm{a}$ & $20.00 \mathrm{~g}$ & $0.00 \mathrm{~h}$ & $20.00^{4}$ & $6.35^{4}$ & $3.52^{4}$ & $1.80^{4}$ & 2 \\
\hline Rangpur & Orange berry & $1 / 5$ & 9 & $100.00 \mathrm{a}$ & $100.00 \mathrm{a}$ & $11.11 \mathrm{~h}$ & $0.00 \mathrm{~h}$ & $20.00^{4}$ & $4.08^{4}$ & $4.92^{4}$ & $0.83^{4}$ & 2 \\
\hline Orange jasmine & Orange berry & $5 / 5$ & 10 & $70.00 \mathrm{e}$ & 10.00h & $0.00 \mathrm{k}$ & $0.00 \mathrm{~h}$ & $20.00^{4,5}$ & $3.00^{4,5}$ & $4.00^{4,5}$ & $0.75^{4,5}$ & 2 \\
\hline Orange jasmine & Hamlin & $5 / 1$ & 30 & $60.00 \mathrm{e}$ & $36.67 \mathrm{e}$ & $0.00 \mathrm{k}$ & $0.00 \mathrm{~h}$ & $18.98^{5}$ & $2.86^{5}$ & $4.80^{5}$ & $0.60^{5}$ & 2 \\
\hline
\end{tabular}


Table 2. Cont.

\begin{tabular}{|c|c|c|c|c|c|c|c|c|c|c|c|c|}
\hline \multirow{2}{*}{ Scion } & \multirow{2}{*}{ Rootstock } & \multirow{2}{*}{$\mathrm{PG}^{1}$} & \multirow{2}{*}{$\mathbf{N}^{*}$} & \multicolumn{4}{|c|}{ MAG } & \multirow{2}{*}{$\begin{array}{c}\mathbf{H} \\
(\mathrm{cm})\end{array}$} & \multirow{2}{*}{$\begin{array}{c}\text { SD } \\
(\mathrm{mm})\end{array}$} & \multirow{2}{*}{$\begin{array}{c}\text { RD } \\
(\mathrm{mm})\end{array}$} & \multirow{2}{*}{ SD/RD } & \multirow{2}{*}{$\mathrm{GC}^{3}$} \\
\hline & & & & 2 & 7 & 14 & $21^{2}$ & & & & & \\
\hline Orange jasmine & Swingle & $5 / 2$ & 30 & $83.33 c$ & $53.33 d$ & $6.67 \mathrm{i}$ & $0.00 \mathrm{~h}$ & $18.00^{4}$ & $3.39^{4}$ & $5.61^{4}$ & $0.63^{4}$ & 2 \\
\hline Orange jasmine & Pineapple & $5 / 1$ & 30 & $20.00 \mathrm{~h}$ & $6.67 i$ & $0.00 \mathrm{k}$ & $0.00 \mathrm{~h}$ & $16.45^{5}$ & $2.25^{5}$ & $3.65^{5}$ & $0.62^{5}$ & 2 \\
\hline Orange jasmine & Sour orange & $5 / 1$ & 30 & $40.00 \mathrm{f}$ & $16.67 \mathrm{~g}$ & $0.00 \mathrm{k}$ & $0.00 \mathrm{~h}$ & $15.20^{5}$ & $2.50^{5}$ & $4.68^{5}$ & $0.55^{5}$ & 2 \\
\hline Orange jasmine & Pera & $5 / 1$ & 15 & $46.67 f$ & $33.33 \mathrm{e}$ & $7.14 \mathrm{i}$ & $0.00 \mathrm{~h}$ & $15.00^{5}$ & $2.06^{5}$ & $3.12^{5}$ & $0.66^{5}$ & 2 \\
\hline Orange jasmine & Ponkan & $5 / 1$ & 30 & $16.67 \mathrm{~h}$ & $16.67 \mathrm{~g}$ & $0.00 \mathrm{k}$ & $0.00 \mathrm{~h}$ & $14.26^{5}$ & $2.59^{5}$ & $4.73^{5}$ & $0.55^{5}$ & 2 \\
\hline Wampee & Tabog & $5 / 4$ & 5 & $100.00 \mathrm{a}$ & $100.00 \mathrm{a}$ & $0.00 \mathrm{k}$ & $0.00 \mathrm{~h}$ & $14.22^{5}$ & $4.67^{5}$ & $3.54^{5}$ & $1.33^{5}$ & 2 \\
\hline Ponkan & Orange jasmine & $1 / 5$ & 30 & $10.00 \mathrm{i}$ & $3.33 \mathrm{j}$ & $0.00 \mathrm{k}$ & $0.00 \mathrm{~h}$ & $14.00^{4,5}$ & $3.00^{4,5}$ & $4.20^{4,5}$ & $0.71^{4,5}$ & 2 \\
\hline Curry tree & Wampee & $5 / 5$ & 5 & $100.00 \mathrm{a}$ & $60.00 \mathrm{~d}$ & $60.00 \mathrm{e}$ & $0.00 \mathrm{~h}$ & $13.33 d$ & $3.86 \mathrm{~d}$ & $4.45 \mathrm{e}$ & $0.86 c$ & 2 \\
\hline Box orange & Orange jasmine & $3 / 5$ & 15 & $46.67 f$ & $6.67 i$ & $0.00 \mathrm{k}$ & $0.00 \mathrm{~h}$ & $13.00^{4,5}$ & $2.40^{4,5}$ & $3.00^{4,5}$ & $0.80^{4,5}$ & 2 \\
\hline Swingle & Orange jasmine & $2 / 5$ & 30 & $40.00 f$ & $3.33 \mathrm{j}$ & $0.00 \mathrm{k}$ & $0.00 \mathrm{~h}$ & $13.00^{4,5}$ & $3.00^{4,5}$ & $4.00^{4,5}$ & $0.75^{4,5}$ & 2 \\
\hline Orange jasmine & Rangpur & $5 / 1$ & 30 & $36.67 f$ & $16.67 \mathrm{~g}$ & $0.00 \mathrm{k}$ & $0.00 \mathrm{~h}$ & $12.70^{5}$ & $2.72^{5}$ & $5.09^{5}$ & $0.53^{5}$ & 2 \\
\hline Orange jasmine & Lemon & $5 / 1$ & 30 & $66.67 \mathrm{e}$ & $43.33 \mathrm{e}$ & $0.00 \mathrm{k}$ & $0.00 \mathrm{~h}$ & $12.57^{5}$ & $2.74^{5}$ & $5.51^{5}$ & $0.50^{5}$ & 2 \\
\hline Orange berry & Curry tree & $5 / 5$ & 1 & $100.00 \mathrm{a}$ & $100.00 \mathrm{a}$ & $0.00 \mathrm{k}$ & $0.00 \mathrm{~h}$ & $10.00^{4,5}$ & $2.00^{4,5}$ & $3.00^{4,5}$ & $0.67^{4,5}$ & 2 \\
\hline Curry tree & Orange jasmine & $5 / 5$ & 25 & $24.00 \mathrm{~g}$ & $4.00 \mathrm{j}$ & $0.00 \mathrm{k}$ & $0.00 \mathrm{~h}$ & $9.20^{4,5}$ & $2.70^{4,5}$ & $0.56^{4,5}$ & $4.82^{4,5}$ & 3 \\
\hline Orange jasmine & Wampee & $5 / 5$ & 18 & $38.89 \mathrm{f}$ & $0.00 \mathrm{k}$ & $0.00 \mathrm{k}$ & $0.00 \mathrm{~h}$ & $\mathrm{~d}^{6}$ & $\mathrm{~d}^{6}$ & $\mathrm{~d}^{6}$ & $\mathrm{~d}^{6}$ & 4 \\
\hline Hamlin & Orange jasmine & $1 / 5$ & 30 & $26.67 \mathrm{~g}$ & $0.00 \mathrm{k}$ & $0.00 \mathrm{k}$ & $0.00 \mathrm{~h}$ & $\mathrm{~d}^{6}$ & $d^{6}$ & $d^{6}$ & $d^{6}$ & 4 \\
\hline Rangpur & Orange jasmine & $1 / 5$ & 30 & $13.33 \mathrm{i}$ & $0.00 \mathrm{k}$ & $0.00 \mathrm{k}$ & $0.00 \mathrm{~h}$ & $\mathrm{~d}^{6}$ & $d^{6}$ & $d^{6}$ & $\mathrm{~d}^{6}$ & 4 \\
\hline Sour orange & Orange jasmine & $1 / 5$ & 30 & $10.00 \mathrm{i}$ & $0.00 \mathrm{k}$ & $0.00 \mathrm{k}$ & $0.00 \mathrm{~h}$ & $\mathrm{~d}^{6}$ & $d^{6}$ & $d^{6}$ & $d^{6}$ & 4 \\
\hline Curry tree & Orange berry & $5 / 5$ & 1 & $0.00 \mathrm{k}$ & $0.00 \mathrm{k}$ & $0.00 \mathrm{k}$ & $0.00 \mathrm{~h}$ & $\mathrm{~d}^{6}$ & $d^{6}$ & $d^{6}$ & $d^{6}$ & 4 \\
\hline Wampee & Orange berry & $5 / 5$ & 1 & $0.00 \mathrm{k}$ & $0.00 \mathrm{k}$ & $0.00 \mathrm{k}$ & $0.00 \mathrm{~h}$ & $d^{6}$ & $d^{6}$ & $d^{6}$ & $\mathrm{~d}^{6}$ & 4 \\
\hline Wampee & Orange jasmine & $5 / 5$ & 18 & $0.00 \mathrm{k}$ & $0.00 \mathrm{k}$ & $0.00 \mathrm{k}$ & $0.00 \mathrm{~h}$ & $\mathrm{~d}^{6}$ & $d^{6}$ & $d^{6}$ & $\mathrm{~d}^{6}$ & 4 \\
\hline Orange berry & Wampee & $5 / 5$ & 1 & $0.00 \mathrm{k}$ & $0.00 \mathrm{k}$ & $0.00 \mathrm{k}$ & $0.00 \mathrm{~h}$ & $\mathrm{~d}^{6}$ & $d^{6}$ & $d^{6}$ & $\mathrm{~d}^{6}$ & 4 \\
\hline Orange berry & Orange jasmine & $5 / 5$ & 10 & $0.00 \mathrm{k}$ & $0.00 \mathrm{k}$ & $0.00 \mathrm{k}$ & $0.00 \mathrm{~h}$ & $d^{6}$ & $d^{6}$ & $d^{6}$ & $d^{6}$ & 4 \\
\hline Orange jasmine & Bael & $5 / 4$ & 8 & $0.00 \mathrm{k}$ & $0.00 \mathrm{k}$ & $0.00 \mathrm{k}$ & $0.00 \mathrm{~h}$ & $\mathrm{~d}^{6}$ & $d^{6}$ & $d^{6}$ & $\mathrm{~d}^{6}$ & 4 \\
\hline Orange jasmine & Curry tree & $5 / 5$ & 25 & $0.00 \mathrm{k}$ & $0.00 \mathrm{k}$ & $0.00 \mathrm{k}$ & $0.00 \mathrm{~h}$ & $\mathrm{~d}^{6}$ & $d^{6}$ & $d^{6}$ & $\mathrm{~d}^{6}$ & 4 \\
\hline Orange jasmine & Rubi & $5 / 1$ & 15 & $0.00 \mathrm{k}$ & $0.00 \mathrm{k}$ & $0.00 \mathrm{k}$ & $0.00 \mathrm{~h}$ & $\mathrm{~d}^{6}$ & $d^{6}$ & $d^{6}$ & $\mathrm{~d}^{6}$ & 4 \\
\hline Pineapple & Orange jasmine & $1 / 5$ & 30 & $0.00 \mathrm{k}$ & $0.00 \mathrm{k}$ & $0.00 \mathrm{k}$ & $0.00 \mathrm{~h}$ & $d^{6}$ & $\mathrm{~d}^{6}$ & $d^{6}$ & $\mathrm{~d}^{6}$ & 4 \\
\hline Pomeroy & Orange jasmine & $2 / 5$ & 30 & $0.00 \mathrm{k}$ & $0.00 \mathrm{k}$ & $0.00 \mathrm{k}$ & $0.00 \mathrm{~h}$ & $\mathrm{~d}^{6}$ & $\mathrm{~d}^{6}$ & $d^{6}$ & $\mathrm{~d}^{6}$ & 4 \\
\hline
\end{tabular}

${ }^{*} \mathrm{~N}=$ Number of treatment replications. Mean values followed by the same letter belong to the same group by the Scott-Knott test (5\%). ${ }^{1}$ Phylogenetic group according to classification presented in Table $1 .{ }^{2}$ Pruned plants (height data not available). ${ }^{3}$ Graft compatibility groups: GC 1, full graft compatibility; GC 2, medium-term incompatibility; GC 3, short-term incompatibility; GC 4, full graft incompatibility. ${ }^{4}$ Data from less than three plants (not statistically analyzed). ${ }^{5} 7$ MAG measures. ${ }^{6}$ Dead plants.

\subsection{Second Group of $S / R$ Combinations (Experiment 2)}

The $\mathrm{S} / \mathrm{R}$ combinations analyzed from the second group showed at least $70 \%$ of grafttake in the first assessment (2 MAG). However, results ranged from $0 \%$ to $100 \%$ in the following assessments (7-12 and 13-16 MAG). Sixteen of the 39 S/R combinations (41\%) showed a minimum of $60 \%$ of graft-take, with the average plant height being higher than $18 \mathrm{~cm}$ (Table 3). The average SD of these combinations ranged from 3.60 to $7.15 \mathrm{~mm}$ for the first four SK clusters. The average RD ranged from 4.08 to $8.39 \mathrm{~mm}$ for the first six SK clusters. The average $\mathrm{SD} / \mathrm{RD}$ relation ranged from 0.77 to 1.73 for the first six SK clusters (Table 3). The best-performing S/R combinations belonged to PGs 1, 2, and 5 (Table 1). Ten of the $39 \mathrm{~S} / \mathrm{R}$ combinations ( $25.6 \%$ ) presented with less than $10 \%$ of graft-take (Table 3 ).

\subsection{Anatomical Evaluation}

Anatomical analyses were conducted to confirm the compatibility of the grafting process with different $S / R$ combinations within Aurantioideae. Figure 1 shows the phenotype of the grafted plants and their anatomical analyses. Phloroglucinol was used to stain the lignified vascular tissues in pink, and the continuity of xylem vessels between the $S / R$ was observed. Furthermore, phenolic compound accumulation was also verified. 
Table 3. Graft-take (\%) of the second group of scion/rootstock combinations within different Aurantioideae phylogenetic groups (PGs) at 2, 7-12, and 13-16 months after grafting (MAG), mean values of plant height $(\mathrm{H})$, scion stem diameter (SD), rootstock stem diameter (RD), scion and rootstock stem diameter relation (SD/RD) at 13-16 MAG, and graft compatibility group (GC).

\begin{tabular}{|c|c|c|c|c|c|c|c|c|c|c|c|}
\hline \multirow{2}{*}{ Scion } & \multirow{2}{*}{ Rootstock } & \multirow{2}{*}{$\mathrm{PG}^{1}$} & \multirow{2}{*}{$\mathbf{N}^{*}$} & \multicolumn{3}{|c|}{ MAG } & \multirow{2}{*}{$\begin{array}{c}\mathbf{H} \\
(\mathrm{cm})\end{array}$} & \multirow{2}{*}{$\begin{array}{c}\text { SD } \\
(\mathrm{mm})\end{array}$} & \multirow{2}{*}{$\begin{array}{c}\text { RD } \\
(\mathrm{mm})\end{array}$} & \multirow{2}{*}{ SD/RD } & \multirow{2}{*}{$\mathrm{GC}^{2}$} \\
\hline & & & & 2 & $7-12$ & $13-16$ & & & & & \\
\hline Rangpur & Rangpur & $1 / 1$ & 10 & $100.00 \mathrm{a}$ & $100.00 \mathrm{a}$ & $100.00 \mathrm{a}$ & $113.30 \mathrm{a}$ & $6.77 a$ & $8.39 a$ & $0.80 \mathrm{f}$ & 1 \\
\hline Swingle & Swingle & $2 / 2$ & 10 & $100.00 \mathrm{a}$ & $100.00 \mathrm{a}$ & $100.00 \mathrm{a}$ & $97.45 b$ & $5.96 b$ & $7.20 \mathrm{~b}$ & $0.83 \mathrm{f}$ & 1 \\
\hline Pomeroy & Pomeroy & $2 / 2$ & 10 & $100.00 \mathrm{a}$ & $100.00 \mathrm{a}$ & $100.00 \mathrm{a}$ & $64.30 \mathrm{c}$ & $4.40 \mathrm{~d}$ & $5.07 \mathrm{e}$ & $0.87 f$ & 2 \\
\hline Orange berry & Box orange & $5 / 3$ & 17 & $100.00 \mathrm{a}$ & $100.00 \mathrm{a}$ & $100.00 \mathrm{a}$ & $39.33 d$ & $4.06 \mathrm{~d}$ & $4.90 \mathrm{e}$ & $0.84 \mathrm{f}$ & 2 \\
\hline Wampee & Wampee & $5 / 5$ & 10 & $100.00 \mathrm{a}$ & $100.00 \mathrm{a}$ & $100.00 \mathrm{a}$ & $28.00 \mathrm{e}$ & $5.15 c$ & $4.97 \mathrm{e}$ & $1.04 \mathrm{e}$ & 2 \\
\hline Wampee & Box orange & $5 / 3$ & 17 & $100.00 \mathrm{a}$ & $100.00 \mathrm{a}$ & $100.00 \mathrm{a}$ & $27.46 \mathrm{e}$ & $6.96 a$ & $5.08 \mathrm{e}$ & $1.39 \mathrm{~b}$ & 2 \\
\hline Curry tree & Wampee & $5 / 5$ & 10 & $100.00 \mathrm{a}$ & $100.00 \mathrm{a}$ & $100.00 \mathrm{a}$ & $20.90 \mathrm{e}$ & $4.06 \mathrm{~d}$ & $4.96 \mathrm{e}$ & $0.83 \mathrm{f}$ & 3 \\
\hline Wampee & Pomeroy & $5 / 2$ & $40-70$ & $95.71 \mathrm{c}$ & $92.86 b$ & $92.50 \mathrm{~b}$ & $20.10 \mathrm{e}$ & $5.83 b$ & $4.74 \mathrm{e}$ & $1.73 c$ & 2 \\
\hline Wampee & Rangpur & $5 / 1$ & 31 & $100.00 \mathrm{a}$ & $90.32 c$ & $90.32 b$ & $32.75 \mathrm{e}$ & $7.15 a$ & $5.40 \mathrm{~d}$ & $1.33 \mathrm{~b}$ & 2 \\
\hline Valencia & Rangpur & $1 / 1$ & 45 & $100.00 \mathrm{a}$ & 91.11c & $90.00 \mathrm{~b}$ & $48.80 \mathrm{~d}$ & $5.64 b$ & $7.31 b$ & $0.77 \mathrm{f}$ & 1 \\
\hline Pomeroy & Wampee & $2 / 5$ & $40-70$ & $91.43 d$ & $88.57 d$ & $87.50 \mathrm{~b}$ & $70.83 c$ & $5.03 c$ & $4.51 \mathrm{f}$ & $1.11 \mathrm{~d}$ & 2 \\
\hline Wampee & Swingle & $5 / 2$ & 70 & $95.00 \mathrm{c}$ & $85.00 \mathrm{~d}$ & $85.00 c$ & $38.03 d$ & $5.62 b$ & $6.54 c$ & $0.87 \mathrm{f}$ & 2 \\
\hline Rangpur & Wampee & $1 / 5$ & 31 & $100.00 \mathrm{a}$ & $100.00 \mathrm{a}$ & $80.65 c$ & $31.67 \mathrm{e}$ & $4.72 \mathrm{c}$ & $4.16 f$ & $1.16 c$ & 3 \\
\hline Swingle & Wampee & $2 / 5$ & 70 & $95.00 \mathrm{c}$ & $80.00 \mathrm{~d}$ & $80.00 c$ & $61.28 \mathrm{c}$ & $5.52 \mathrm{~b}$ & $5.15 \mathrm{e}$ & $1.08 \mathrm{~d}$ & 2 \\
\hline Curry tree & Curry tree & $5 / 5$ & 10 & $80.00 \mathrm{e}$ & $70.00 \mathrm{e}$ & $70.00 \mathrm{~d}$ & $71.00 \mathrm{c}$ & $6.13 b$ & $6.57 c$ & $0.93 e$ & 1 \\
\hline Orange berry & Pomeroy & $5 / 2$ & 70 & $95.00 \mathrm{c}$ & $95.00 \mathrm{~b}$ & $60.00 \mathrm{~d}$ & $18.92 \mathrm{e}$ & $3.60 \mathrm{~d}$ & $4.08 \mathrm{f}$ & $0.88 \mathrm{f}$ & 3 \\
\hline Valencia & Wampee & $1 / 5$ & 70 & $98.57 b$ & $87.14 d$ & $57.14 d$ & $22.73 \mathrm{e}$ & $4.37 \mathrm{~d}$ & $3.99 \mathrm{f}$ & $1.10 \mathrm{~d}$ & 3 \\
\hline Tabog & Wampee & $4 / 5$ & 6 & $100.00 \mathrm{a}$ & $100.00 \mathrm{a}$ & $50.00 \mathrm{e}$ & $35.83 d$ & $3.98 \mathrm{~d}$ & $4.13 \mathrm{f}$ & $0.97 e$ & 3 \\
\hline Box orange & Wampee & $3 / 5$ & 17 & $100.00 \mathrm{a}$ & 100.00 & $50.00 \mathrm{e}$ & $31.08 \mathrm{e}$ & $4.32 \mathrm{~d}$ & $3.75 f$ & $1.20 c$ & 3 \\
\hline Orange berry & Rangpur & $5 / 1$ & 70 & $85.00 \mathrm{~d}$ & $75.00 \mathrm{e}$ & $50.00 \mathrm{e}$ & $27.55 \mathrm{e}$ & $4.04 \mathrm{~d}$ & $5.07 \mathrm{e}$ & $0.80 \mathrm{f}$ & 3 \\
\hline Pomeroy & Curry tree & $2 / 5$ & $40-50$ & $90.00 \mathrm{~d}$ & $75.00 \mathrm{e}$ & $46.00 \mathrm{e}$ & $73.69 c$ & $5.86 \mathrm{~b}$ & $4.35 \mathrm{f}$ & $1.36 \mathrm{~b}$ & 2 \\
\hline Swingle & Curry tree & $2 / 5$ & 10 & $90.00 \mathrm{~d}$ & $70.00 \mathrm{e}$ & $40.00 \mathrm{e}$ & $45.13 d$ & $7.45 a$ & $4.13 \mathrm{f}$ & $1.80 \mathrm{a}$ & 4 \\
\hline Curry tree & Rangpur & $5 / 1$ & $10-70$ & $90.00 \mathrm{~d}$ & $65.00 \mathrm{e}$ & $40.00 \mathrm{e}$ & $24.25 \mathrm{e}$ & $4.40 \mathrm{~d}$ & $5.84 \mathrm{~d}$ & $0.75 f$ & 3 \\
\hline Curry tree & Pomeroy & $5 / 2$ & $40-50$ & $100.00 \mathrm{a}$ & $82.00 \mathrm{~d}$ & $30.00 \mathrm{e}$ & $20.56 \mathrm{e}$ & $4.05 \mathrm{~d}$ & $5.68 \mathrm{~d}$ & $0.72 \mathrm{f}$ & 3 \\
\hline Rangpur & Curry tree & $1 / 5$ & $10-70$ & $100.00 \mathrm{a}$ & $55.00 \mathrm{f}$ & $20.00 \mathrm{f}$ & $41.00^{3}$ & $5.77^{3}$ & $4.07^{3}$ & $1.43^{3}$ & 4 \\
\hline Wampee & Curry tree & $5 / 5$ & 10 & $100.00 \mathrm{a}$ & $90.00 \mathrm{c}$ & $20.00 \mathrm{f}$ & $23.50^{3}$ & $6.84^{3}$ & $3.85^{3}$ & $1.77^{3}$ & 4 \\
\hline Wampee & Tabog & $5 / 4$ & 6 & $100.00 \mathrm{a}$ & $83.33 d$ & $16.67 f$ & $37.00^{3}$ & $6.47^{3}$ & $4.13^{3}$ & $1.57^{3}$ & 4 \\
\hline Rangpur & Orange berry & $1 / 5$ & 70 & $95.00 \mathrm{c}$ & $70.00 \mathrm{e}$ & $10.00 \mathrm{~g}$ & $37.25^{3}$ & $5.09^{3}$ & $4.84^{3}$ & $1.06^{3}$ & 3 \\
\hline Valencia & Curry tree & $1 / 5$ & $10-70$ & $80.00 \mathrm{e}$ & $50.00 \mathrm{f}$ & $10.00 \mathrm{~g}$ & $32.00^{3}$ & $4.12^{3}$ & $4.26^{3}$ & $0.97^{3}$ & 3 \\
\hline Orange berry & Swingle & $5 / 2$ & 70 & $90.00 \mathrm{~d}$ & $70.00 \mathrm{e}$ & $10.00 \mathrm{~g}$ & $23.00^{3}$ & $3.65^{3}$ & $4.65^{3}$ & $0.79^{3}$ & 3 \\
\hline Box orange & Orange berry & $3 / 5$ & 17 & $91.67 \mathrm{~d}$ & $33.33 \mathrm{~g}$ & $8.33 \mathrm{~g}$ & $46.00^{3}$ & $5.58^{3}$ & $4.11^{3}$ & $1.36^{3}$ & 4 \\
\hline Curry tree & Swingle & $5 / 2$ & 10 & $100.00 \mathrm{a}$ & $100.00 \mathrm{a}$ & $0.00 \mathrm{~h}$ & $21.45^{4}$ & $3.96^{4}$ & $5.90^{4}$ & $0.68^{4}$ & 3 \\
\hline Curry tree & Orange berry & $5 / 5$ & 10 & $100.00 \mathrm{a}$ & $60.00 \mathrm{e}$ & $0.00 \mathrm{~h}$ & $15.92^{4}$ & $3.05^{4}$ & $3.16^{4}$ & $0.97^{4}$ & 3 \\
\hline Orange berry & Curry tree & $5 / 5$ & $6-10$ & $90.00 \mathrm{~d}$ & $50.00 \mathrm{f}$ & $0.00 \mathrm{~h}$ & $18.20^{4}$ & $2.70^{4}$ & $3.20^{4}$ & $0.84^{4}$ & 3 \\
\hline Valencia & Orange berry & $1 / 5$ & 70 & $80.00 \mathrm{e}$ & $65.00 \mathrm{e}$ & $0.00 \mathrm{~h}$ & $27.85^{4}$ & $4.25^{4}$ & $4.22^{4}$ & $1.02^{4}$ & 3 \\
\hline Pomeroy & Orange berry & $2 / 5$ & 70 & $65.00 \mathrm{f}$ & $25.00 \mathrm{~g}$ & $0.00 \mathrm{~h}$ & $25.92^{4}$ & $4.03^{4}$ & $4.07^{4}$ & $1.00^{4}$ & 3 \\
\hline Swingle & Orange berry & $2 / 5$ & 70 & $60.00 f$ & $20.00 \mathrm{~g}$ & $0.00 \mathrm{~h}$ & $30.63^{4}$ & $4.85^{4}$ & $4.66^{4}$ & $1.05^{4}$ & 3 \\
\hline Valencia & Pomeroy & $1 / 2$ & 70 & $90.00 d$ & $85.00 \mathrm{~d}$ & NA & $58.20^{4}$ & $5.73^{4}$ & $7.75^{4}$ & $0.73^{4}$ & 1 \\
\hline Valencia & Bael & $1 / 4$ & 10 & $70.00 \mathrm{~g}$ & $0.00 \mathrm{~h}$ & $0.00 \mathrm{~h}$ & $10.50^{5}$ & $2.20^{5}$ & $6.25^{5}$ & $0.37^{5}$ & 3 \\
\hline
\end{tabular}

${ }^{*} \mathrm{~N}=$ Number of treatment replications. Mean values followed by the same letter belong to the same group by the Scott-Knott test (5\%). ${ }^{1}$ Phylogenetic group according to classification presented in Table $1 .{ }^{2}$ Graft compatibility groups: GC 1, full graft compatibility; GC 2, potential graft compatibility; GC 3, partial graft incompatibility; GC 4, full graft incompatibility. ${ }^{3}$ Data from less than three plants. ${ }^{4}$ Measurement performed at 7-12 MAG. ${ }^{5}$ Measurement performed at 2 MAG. NA: not assessed.

Full vascular connection was also observed in both heterogenous combinations, which varied within different PGs. This included varieties such as Valencia/Rangpur (Figure 1a-d), Valencia/Pomeroy (Figure 1e-h), and Swingle/Wampee (Figure 1i-l), as well as homogenous autograft combinations such as Rangpur/Rangpur (Figure 2a-d), Swingle/Swingle (Figure 2e-h), Pomeroy/Pomeroy (Figure 2i-l), Wampee/Wampee (Figure 2q-t), and Curry tree/Curry tree (Figure $3 \mathrm{i}-\mathrm{l}$ ), or reciprocal graft combinations such as Wampee/Swingle (Figure 3e-h). Within all these combinations, the xylem connection had a high amplitude, with phenolic compound accumulation not being observed. 


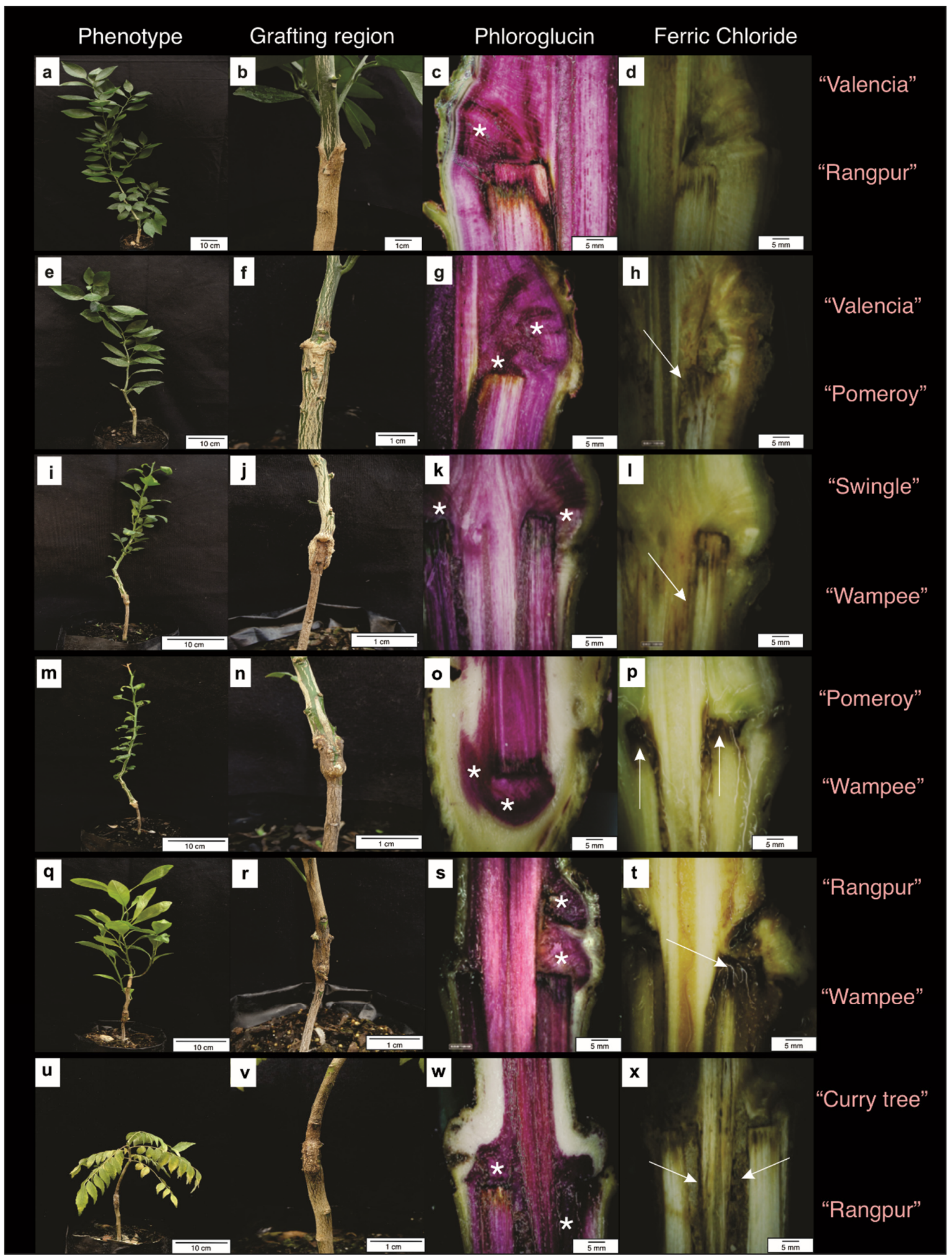

Figure 1. Six grafting combinations of Aurantioideae plants indicating the plant phenotype $(\mathbf{a}, \mathbf{e}, \mathbf{i}, \mathbf{m}, \mathbf{q}, \mathbf{u})$ and images of the graft union $(\mathbf{b}, \mathbf{f}, \mathbf{j}, \mathbf{n}, \mathbf{r}, \mathbf{v})$, phloroglucin reaction $(\mathbf{c}, \mathbf{g}, \mathbf{k}, \mathbf{o}, \mathbf{s}, \mathbf{w})$, and ferric chloride $(\mathbf{d}, \mathbf{h}, \mathbf{l}, \mathbf{p}, \mathbf{t}, \mathbf{x})$. Asterisks in $(\mathbf{c}, \mathbf{g}, \mathbf{k}, \mathbf{o}, \mathbf{s}, \mathbf{w})$ : phloroglucin reaction in pink indicates lignified cell walls. Arrows in $(\mathbf{h}, \mathbf{l}, \mathbf{p}, \mathbf{t}, \mathbf{x})$ : ferric chloride indicates phenolic compounds in brown. Valencia/Rangpur does not indicate phenolic compounds in the grafted region. Strong reactions were observed in Rangpur/Wampee in the grafted region (arrows). 


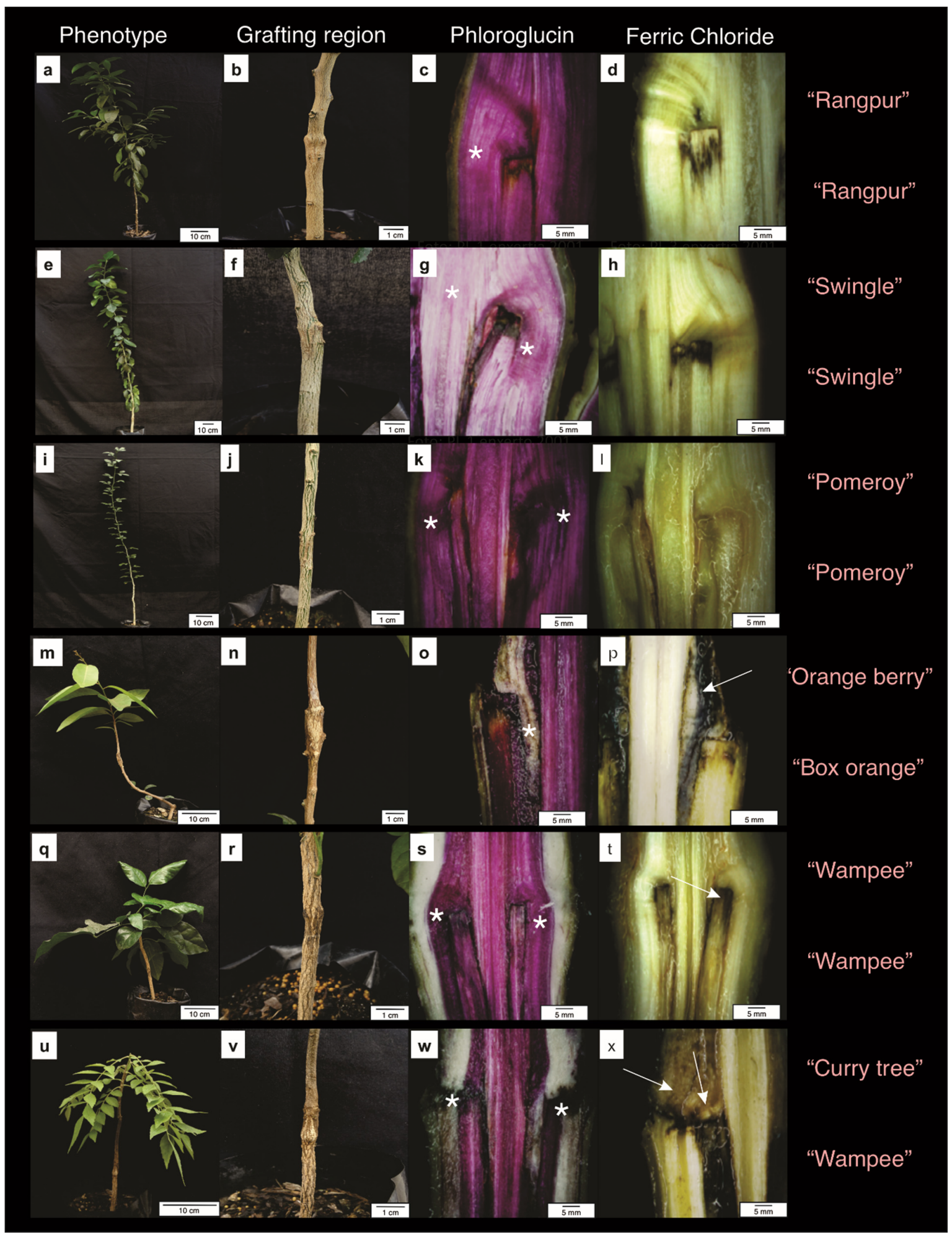

Figure 2. Six grafting combinations of Aurantioideae plants indicating the plant phenotype $(\mathbf{a}, \mathbf{e}, \mathbf{i}, \mathbf{m}, \mathbf{q}, \mathbf{u})$, and images of the graft union $(\mathbf{b}, \mathbf{f}, \mathbf{j}, \mathbf{n}, \mathbf{r}, \mathbf{v})$, phloroglucin reaction $(\mathbf{c}, \mathbf{g}, \mathbf{k}, \mathbf{o}, \mathbf{s}, \mathbf{w})$, and ferric chloride $(\mathbf{d}, \mathbf{h}, \mathbf{l}, \mathbf{p}, \mathbf{t}, \mathbf{x})$. Asterisks in $(\mathbf{c}, \mathbf{g}, \mathbf{k}, \mathbf{0}, \mathbf{s}, \mathbf{w})$ : pink indicates lignified cell walls. Arrows in $(\mathbf{p}, \mathbf{t}, \mathbf{x})$ : ferric chloride indicates phenolic compounds in brown. Rangpur/Rangpur, Swingle/Swingle, Wampee/Wampee, and Pomeroy/Pomeroy have great vascular connection $(\mathbf{c}, \mathbf{g}, \mathbf{k}, \mathbf{s})$ and do not present phenolic compounds $(\mathbf{d}, \mathbf{h}, \mathbf{l}, \mathbf{t})$. Orange berry/Box orange and Curry tree/Wampee present a poor vascular connection and phenolic compounds at the graft region. 


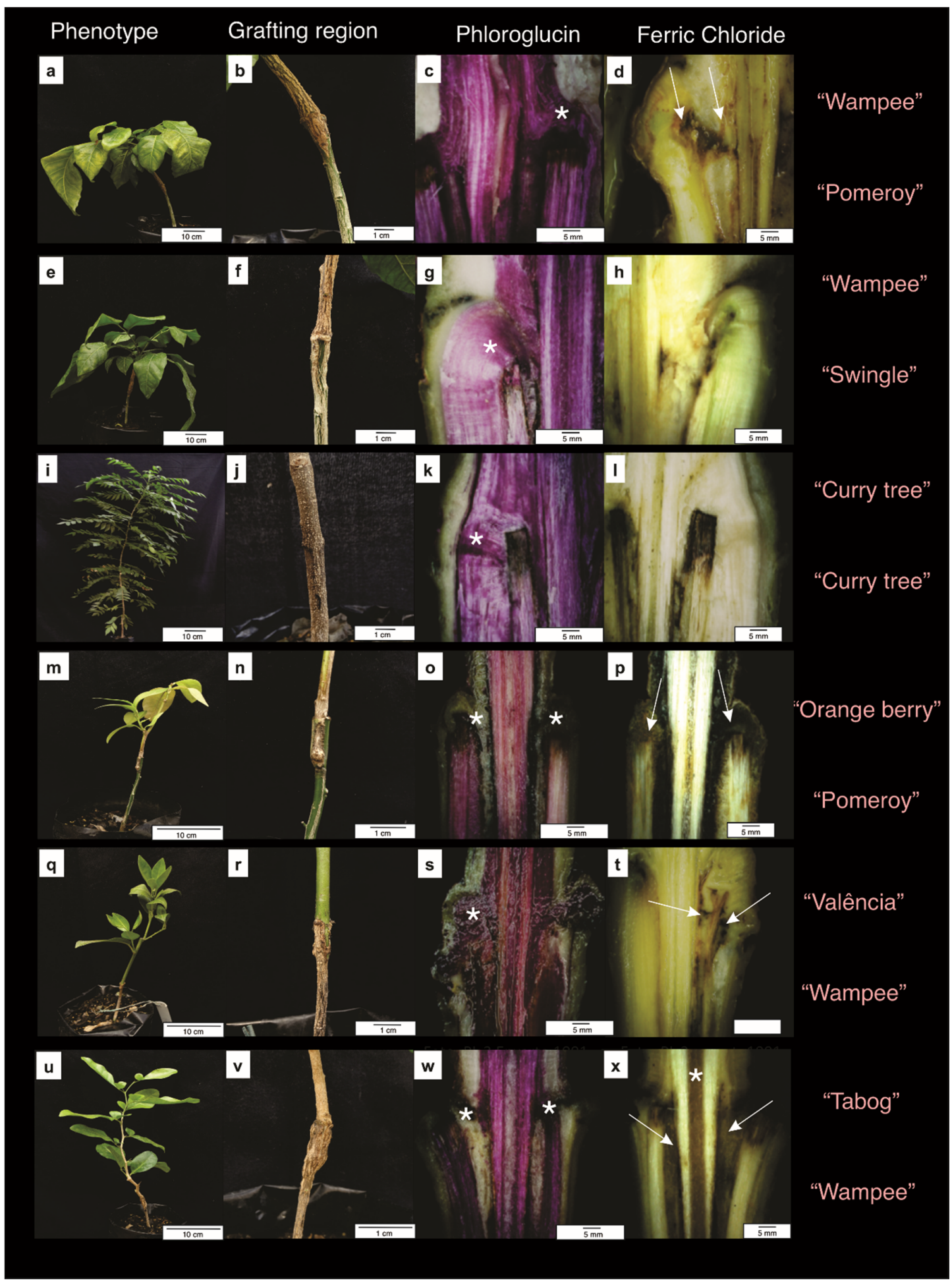

Figure 3. Six grafting combinations of Aurantioideae plants indicating the plant phenotype $(\mathbf{a}, \mathbf{e}, \mathbf{i}, \mathbf{m}, \mathbf{q}, \mathbf{u})$, and images of the graft union $(\mathbf{b}, \mathbf{f}, \mathbf{j}, \mathbf{n}, \mathbf{r}, \mathbf{v})$, phloroglucin reaction $(\mathbf{c}, \mathbf{g}, \mathbf{k}, \mathbf{o}, \mathbf{s}, \mathbf{w})$, and ferric chloride $(\mathbf{d}, \mathbf{h}, \mathbf{l}, \mathbf{p}, \mathbf{t}, \mathbf{x})$. Asterisks in $(\mathbf{c}, \mathbf{g}, \mathbf{k}, \mathbf{o}, \mathbf{s}, \mathbf{w})$ : phloroglucin reaction in pink indicates lignified cell walls. Arrows in $(\mathbf{d}, \mathbf{p}, \mathbf{t}, \mathbf{x})$ : ferric chloride indicates phenolic compounds in brown. Wampee/Pomeroy, Wampee/Swingle, and Curry tree/Curry tree have great vascular connection $(\mathbf{c}, \mathbf{g}, \mathbf{k})$ and do present few spots with phenolic compounds $(\mathbf{d}, \mathbf{h}, \mathbf{l})$. Orange berry/Pomeroy, Rangpur/Wampee, and Tabog/Wampee present a poor vascular connection and phenolic compounds at the graft region. The asterisks in (x) indicate phenolic compounds in the pith region of the scion. 
Conversely, partial or fully incompatible $S / R$ combinations were observed in several cases, notably in Rangpur/Wampee (Figure 1q-t), Curry tree/Rangpur (Figure 2u-x), Orange berry/Rangpur (Figure 4a-d), Curry tree/Wampee (Figure 2u-x), Tabog/Wampee (Figure $3 \mathrm{u}-\mathrm{x}$ ), Pomeroy /Curry tree (Figure $4 \mathrm{e}-\mathrm{h}$ ), Curry tree/Pomeroy (Figure $4 \mathrm{i}-\mathrm{l}$ ), and Rangpur/Orange berry (Figure $4 \mathrm{q}-\mathrm{t}$ ). These examples did not have differentiated xylemderived calli and presented with increased levels of phenolic compounds as biochemical markers of graft incompatibility [24-26].

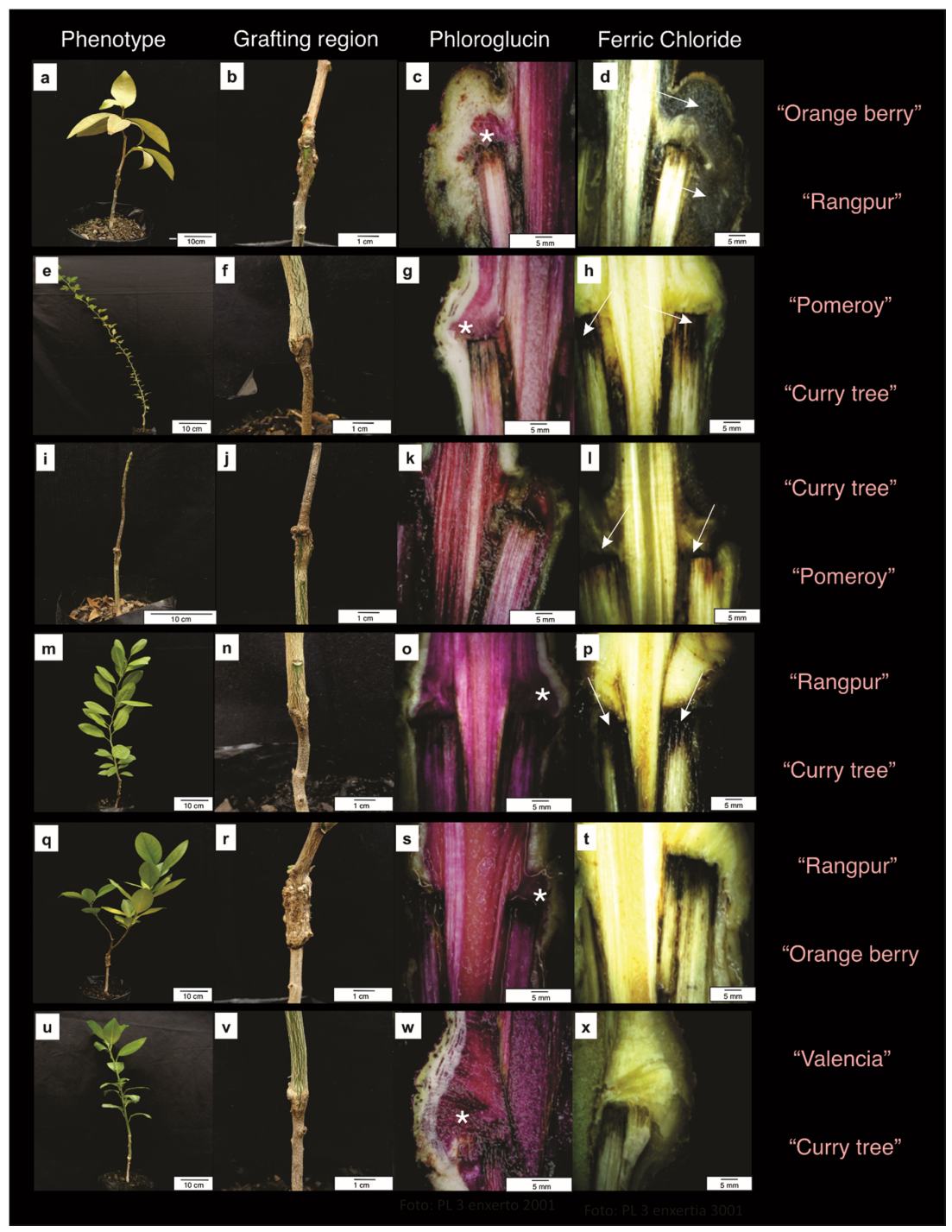

Figure 4. Six grafting combinations of Aurantioideae plants indicating the plant phenotype $(\mathbf{a}, \mathbf{e}, \mathbf{i}, \mathbf{m}, \mathbf{q}, \mathbf{u})$, and images of the graft union $(\mathbf{b}, \mathbf{f}, \mathbf{j}, \mathbf{n}, \mathbf{r}, \mathbf{v})$, phloroglucin reaction $(\mathbf{c}, \mathbf{g}, \mathbf{k}, \mathbf{o}, \mathbf{s}, \mathbf{w})$, and ferric chloride $(\mathbf{d}, \mathbf{h}, \mathbf{l}, \mathbf{p}, \mathbf{t}, \mathbf{x})$. Asterisks in $(\mathbf{c}, \mathbf{g}, \mathbf{o}, \mathbf{s}, \mathbf{w})$ : phloroglucin reaction in pink indicates lignified cell walls. Arrows in $(\mathbf{d}, \mathbf{h}, \mathbf{l}, \mathbf{p})$ : ferric chloride indicates phenolic compounds in brown. Among those combinations, only Valencia/Curry tree presents some vascular connection $\left({ }^{*}\right.$ in $\left.\mathbf{w}\right)$, whereas the others do not present a full connection $\left({ }^{*}\right.$ in $\left.(\mathbf{c}, \mathbf{g}, \mathbf{o}, \mathbf{s})\right)$. Phenolic compounds at the grafting region were abundant in Orange berry/Rangpur (d), Pomeroy/Curry tree (h), Curry tree/Pomeroy (l), and Rangpur/Curry tree (p).

\subsection{Field Assessment and CLas Detection (Experiment 3)}

Seven of the $10 \mathrm{~S} / \mathrm{R}$ combinations that were evaluated had all replicates still alive 12 months after being transplanted (MAT) into the field. Average plant height ranged from $78 \mathrm{~cm}$ (Box orange/Tabog, 3/4 PGs) to $174 \mathrm{~cm}$ (Hamlin/Rangpur, 1/1 PG) (Table 4). The lowest survival rates were observed for Box orange/Hamlin (3/1 PGs), Rangpur/Bael 
(1/4 PGs), and Rangpur/Wampee (1/5 PGs) with $66.67 \%, 50.00 \%$, and $33.33 \%$, respectively. The average SD of the $10 \mathrm{~S} / \mathrm{R}$ combinations ranged from $20.33 \mathrm{~mm}$ to $30.50 \mathrm{~mm}$ with two SK clusters. The average RD ranged from $21.58 \mathrm{~mm}$ to $42.14 \mathrm{~mm}$ for two SK clusters. The average SD/RD relation ranged from 0.74 to 1.09 , with a non-significant difference (Table 4). All plants from all S/R combinations were HLB-asymptomatic at 12 MAT. However, qPCR analysis for all the plants indicated a 50\% HLB incidence in the Hamlin/Rangpur combination (mean $\mathrm{Ct}=34.35$ ). The other plants were found to be CLas-free, with a Ct higher than 35.00 or with "undetected" status (Table 4).

Table 4. Plant survival percentage of scion/rootstock combinations within different Aurantioideae phylogenetic groups (PGs), and mean values of plant height $(\mathrm{H})$, scion stem diameter (SD), rootstock stem diameter (RD), scion and rootstock stem diameter relation (SD/RD), Huanglongbing (HLB) incidence, and 'Candidatus Liberibacter asiaticus' Ct values ( $\mathrm{Ct}$ ) at 12 months after transplanting to the field, and graft compatibility group (GC).

\begin{tabular}{|c|c|c|c|c|c|c|c|c|c|c|c|}
\hline Scion & Rootstock & $\mathrm{PG}^{1}$ & $\mathbf{N}$ & $\begin{array}{c}\text { Survival }^{2} \\
(\%)\end{array}$ & $\begin{array}{c}\mathrm{H}^{2} \\
(\mathrm{~cm})\end{array}$ & $\begin{array}{l}\mathrm{SD}^{2} \\
(\mathrm{~mm})\end{array}$ & $\begin{array}{l}\mathrm{RD}^{2} \\
(\mathrm{~mm})\end{array}$ & $\mathrm{SD} / \mathrm{RD}^{3}$ & $\begin{array}{c}\text { HLB } \\
(\%)\end{array}$ & Mean Ct & $\mathrm{GC}^{5}$ \\
\hline Hamlin & Rangpur & $1 / 1$ & 10 & $100.00 a$ & $174.20 \mathrm{a}$ & $30.50 \mathrm{a}$ & $41.06 \mathrm{a}$ & 0.75 & 50.00 & 34.35 & 1 \\
\hline Tabog & Rangpur & $4 / 1$ & 9 & 100.00a & $145.22 b$ & $30.02 a$ & $42.14 a$ & 0.74 & 0.00 & 37.46 & 1 \\
\hline Hamlin & Box orange & $1 / 3$ & 10 & $100.00 \mathrm{a}$ & $132.00 \mathrm{~b}$ & $23.71 b$ & $28.39 b$ & 0.84 & 0.00 & $38.44^{6}$ & 2 \\
\hline Tabog & Box orange & $4 / 3$ & 9 & $100.00 \mathrm{a}$ & $120.00 \mathrm{c}$ & $21.81 b$ & $28.76 b$ & 0.77 & 0.00 & 35.66 & 2 \\
\hline Rangpur & Tabog & $1 / 4$ & 9 & $100.00 \mathrm{a}$ & $104.56 c$ & $21.00 \mathrm{~b}$ & $27.39 b$ & 0.83 & 0.00 & 38.06 & 2 \\
\hline Wampee & Rangpur & $5 / 1$ & 8 & $100.00 a$ & $84.63 d$ & $23.19 b$ & $25.97 b$ & 0.90 & 0.00 & 37.28 & 2 \\
\hline Box orange & Tabog & $3 / 4$ & 9 & $100.00 a$ & $78.22 \mathrm{~d}$ & $20.86 b$ & $25.06 b$ & 0.83 & 0.00 & Und $^{7}$ & 2 \\
\hline Box orange & Hamlin & $3 / 1$ & 9 & $66.67 \mathrm{~b}$ & $72.83 \mathrm{~d}$ & $22.54 b$ & $29.10 b$ & 0.77 & 0.00 & Und $^{7}$ & 3 \\
\hline Rangpur & Bael & $1 / 4$ & 4 & $50.00 \mathrm{~b}$ & $112.00 \mathrm{c}$ & $23.18 b$ & $32.52 b$ & 0.76 & 0.00 & 37.16 & 3 \\
\hline Rangpur & Wampee & $1 / 5$ & 6 & $33.33 c$ & $112.00 \mathrm{c}$ & $20.33 b$ & $21.58 b$ & 1.09 & 0.00 & Und $^{7}$ & 4 \\
\hline
\end{tabular}

${ }^{1}$ Phylogenetic group according to classification presented in Table $1 .{ }^{2}$ Mean values followed by the same letter belong to the same group by Scott-Knott test $(5 \%) .{ }^{3}$ Non-significant at Kruskal-Wallis $(5 \%) .{ }^{4}$ Plants with Ct values less than 35.00 were considered CLas-positive. ${ }^{5}$ Graft compatibility groups: GC 1, full graft compatibility; GC 2, potential graft compatibility; GC 3, partial graft incompatibility; GC 4, full graft incompatibility. ${ }^{6}$ Data from one plant. Other replicates were "undetected". ${ }^{7}$ Undetected.

\subsection{Graft Compatibility Classification Using PCA}

Hierarchical clustering analysis was applied to the entire dataset. All evaluated groups of grafted S/R combinations, both in the greenhouse and in the field, were arranged into four clusters (Figure 5), with the biological significance of this varying slightly for each assessment group. For this reason, each cluster for each experiment was presented separately.

For the first group of grafted plants (Experiment 1), the principal component 1 (PC1) and PC2 collectively contributed to $86.1 \%$ of the total variability. The Kaiser criterion was used to choose the two principal components, with eigenvalues of 3.36 for PC1 and 1.02 for PC2. PC1 explained $67.3 \%$ of the variance (Figure $5 \mathrm{a}$ ). Plant height and SD best correlated with this component, with $\mathrm{r}$ values of 0.89 and 0.88 , respectively. PC2 explained $18.8 \%$ of the variance (Figure 5a), with a stronger correlation being observed between SD and $\mathrm{RD}(0.75)$. With findings complementing the cluster analysis, results from the PCA biplot indicated that plant height was positively associated with the percentage of graft-take. This was confirmed by findings from the Pearson correlation analysis $(r=0.75 ; p<0.001)$. This indicated higher growth and development among the most compatible combinations. Based on the results of the PCA from the first experiment, the graft compatibility for the first group was classified as GC 1 for full graft compatibility, including combinations with high graft-take and vegetative growth (e.g., Hamlin/Rangpur and Rangpur/Tabog); GC 2 for medium-term incompatibility, wherein combinations had good initial grafttake but exhibited low survival rates after 7 MAG (e.g., Tabog/Wampee and Orange jasmine/Pomeroy); GC 3 for short-term incompatibility, wherein combinations showed low survival rates after transplantation into the greenhouse (e.g., Curry tree/Orange jasmine); and GC 4 for full graft incompatibility, wherein death occurred in the first 60 days after grafting (e.g., Orange jasmine/Wampee, and Hamlin/Orange jasmine). 


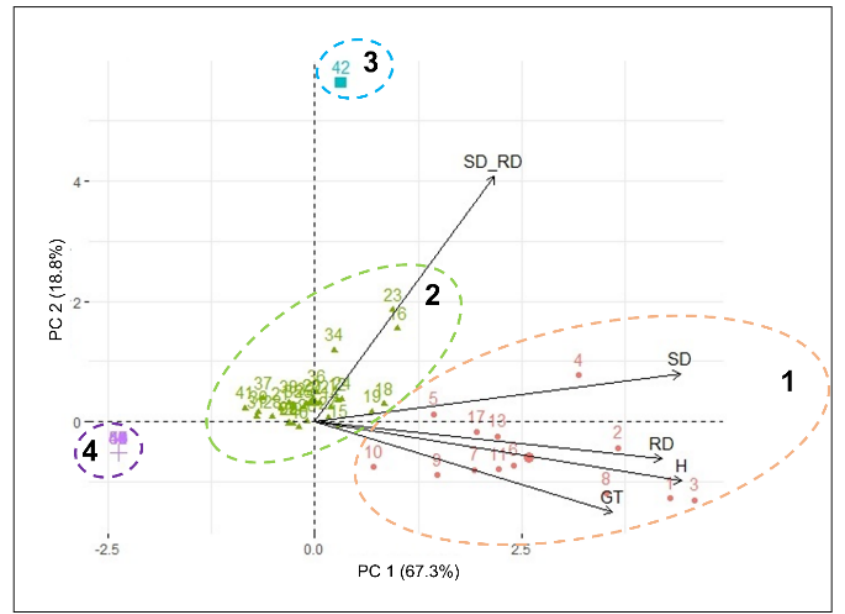

(a)

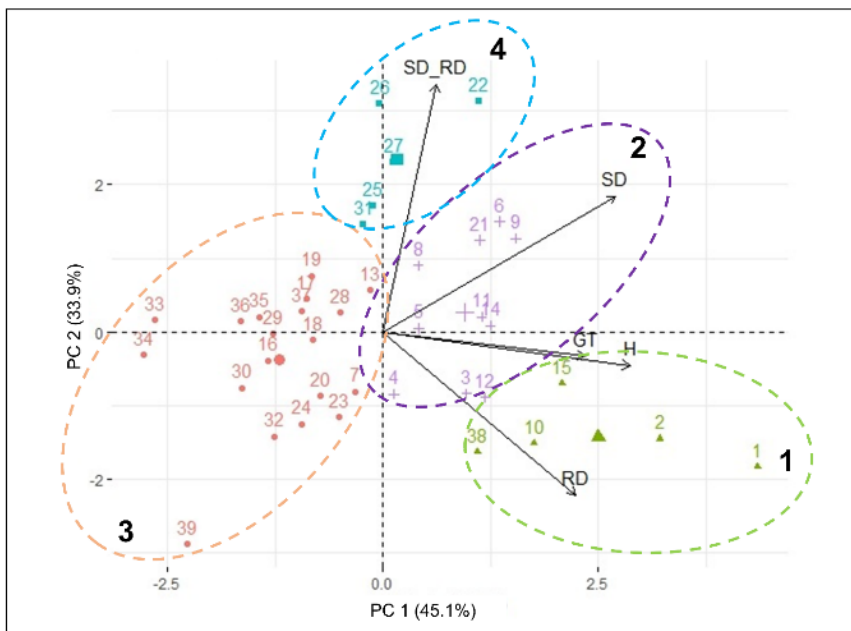

(b)

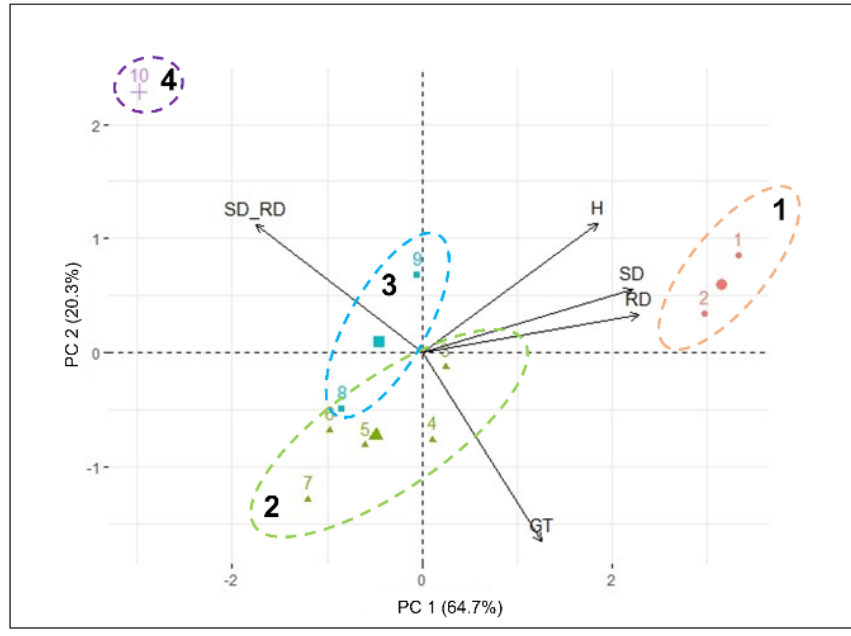

(c)

Figure 5. Principal component analysis (PCA) with principal component 1 and 2 (PC 1 and PC2). (a) PCA indicating the four clusters of the first scion/rootstock combination group. (b) PCA indicating the four clusters of the second scion/rootstock combination group. (c) PCA indicating the four clusters of the field scion/rootstock combination group. The variables used in the analysis were mean values of plant height $(\mathrm{H})$, scion stem diameter (SD), rootstock stem diameter (RD), scion and rootstock stem diameter relation (SD_RD), and graft-take (GT). 
In Experiment 2, the cumulative contribution of the first two PCs explained $79.0 \%$ of the total variability. The eigenvalues were 2.25 for PC1 and 1.69 for PC2. PC1 explained $45.1 \%$ of the total variance (Figure $5 \mathrm{~b}$ ). As was the case in the first group, plant height and SD were best correlated with this component, with $r$ values of 0.70 and 0.62 , respectively. PC2 explained $33.9 \%$ of the variance (Figure $5 b)$. The relation between SD and RD (0.96) and RD alone (0.42) had the closest correlation. The PCA biplot also indicated an association between plant height and the percentage of graft-take. As was the case in the first group, a positive correlation was confirmed using a Pearson correlation analysis $(r=0.73 ; p<0.001)$. Based on the results of the PCA, the graft compatibility for Experiment 2 was classified as GC 1 for full graft compatibility, including combinations with high graft-take and vegetative growth (e.g., Valencia/Rangpur and most autografts); GC 2 for potential graft compatibility, including combinations with high graft-take but less vegetative growth (e.g., Orange berry/Box orange and some autografts); GC 3 for partial graft incompatibility, which had good initial graft-take but low survival at 12 MAG or poor plant growth (e.g., Orange berry/Pomeroy and Valencia/Wampee); and GC 4 for full graft incompatibility, with high initial graft-take but low survival at $12 \mathrm{MAG}$, and a high difference in SD/RD relation (e.g., Swingle/Curry tree and Rangpur/Curry tree).

Under field conditions in Experiment 3, the first two PCs explained 85.0\% of the total data variability, with eigenvalues of 3.23 for the PC1 and 1.01 for the PC2. Results from the PC1 explained $64.7 \%$ of the variance (Figure 5c). RD and SD were better correlated for this component, with $\mathrm{r}$ values of 0.93 and 0.87 , respectively. PC2 explained $20.3 \%$ of the variance (Figure $5 c)$, with results from the graft-take (0.49) and plant height $(0.23)$ having the best correlation. Based on the results of the PCA, the graft-compatibility for the field experiment was classified as follows: GC 1 for full graft compatibility, including combinations with high graft-take and vegetative growth (e.g., Hamlin/Rangpur and Tabog/Rangpur); GC 2 for potential graft compatibility, including combinations with high graft-take but less vegetative growth (e.g., Hamlin/Box orange and Tabog/Box orange); GC 3 for partial graft incompatibility, with low survival and less vegetative growth (e.g., Box orange/Hamlin and Rangpur/Bael); and GC 4 for full graft incompatibility, including plants with low survival rates and severe nutritional deficiency symptoms (e.g., Rangpur/Wampee).

\section{Discussion}

Obtaining graft-compatible combinations may allow exploration of traits of interest both from citrus and citrus relatives within the subfamily Aurantioideae. These may be directly used as rootstocks to bypass biotic and abiotic problems faced in the field with commercially available scion/rootstock combinations. There is also potential for them to be introduced into citrus breeding programs. PCAs were used to group graftcompatible combinations using biometric variables. It was evident that the graft-take rate and plant height were more relevant than the scion/rootstock diameter correlation. Biometric variables and visual inspection of the grafting region have been used in citrus to classify graft compatibility [31,57], but compatible species do not always have a similar scion and rootstock stem diameter [47]. Overall, four compatibility groups were defined in this study: GC 1, full graft compatibility; GC 2, potential graft compatibility; GC 3, partial graft incompatibility; and GC 4, full graft incompatibility (Figure 5).

Grafting between genera from the same botanical family is rarely compatible [32]. In this study, $77 \%$ of the combinations evaluated were found to be fully graft-incompatible. This indicates that taxonomic distance and genetic relations are relevant factors for graft compatibility within Aurantioideae. However, from all PGs tested, some intergeneric combinations showed a high rate of compatibility with Citrus ( $>60 \%$ of graft-take after 12 months) and had suitable levels of plant growth, which comprised up to $20 \%$ of all evaluated combinations. This was notably the case for the Swinglea, Atalantia, and Clausena genera, corroborating previous results from the same genotypes being grafted onto other citrus rootstocks $[13,14,17,18]$. Even some distant combinations such as Orange berry/Box orange survived well, regardless of apparently high levels of phenolic compound accumu- 
lation at the graft union. These results suggest that the graft affinity is highly specific to the combined genotypes. In addition, modern hybridization techniques, such as the "tetrazyg" breeding strategy [70] and protoplast fusion [71,72], have allowed tetraploid rootstocks to be obtained by combining genomes of four phylogenetically distinct varieties. As a result, such somatic hybrids can be both HLB-resistant and more prone to grafting with citrus. Despite the various study opportunities that these new hybridization tools bring, there is still a need to further evaluate early graft compatibility for these new genotypes. Staining techniques such as phloroglucin and ferric chloride methods [58] used in this study may contribute to the rapid prediction of the incompatibility phenomenon [73].

In the first experiment, Orange jasmine (Murraya paniculata) was used as the grafting reference with most Aurantioideae species. This aimed at the exploration of its rusticity and transient multiplication of CLas [33]. However, these plants showed low graft-take for both intrageneric and intergeneric species either in autografts (Table 2; Figure S1). Some genotypes are difficult to graft, even for intraspecific and autografts. This is considered to be the case for many different reasons, from endogenous aspects to environmental conditions [74-76]. Interestingly, Orange jasmine was found to have better performance as a scion, although it has been previously reported to be incompatible with Citrus [17,18]. Nevertheless, despite the low graft-take and subsequent plant growth, a few combinations such as Orange jasmine/Orange jasmine, Orange jasmine/Pomeroy, Orange jasmine/Pera, Orange jasmine/Swingle, Rubi/Orange jasmine, and Tabog/Orange jasmine still had plants that were alive 14 months after being grafted (Table 2). Other combinations also showed very distinct graft compatibility results when they were used as scion or rootstocks, such as Wampee/Rangpur (Figure S2), Orange jasmine/Pomeroy, Rangpur/Bael, and Tabog/Wampee. However, anatomical studies did not show clear differences in graft position. In another study, it was observed that Wampee had a better graft-take rate when used as a scion for citrus rather than as a rootstock [18]. Therefore, in reciprocal grafting between two phylogenetically distinct genotypes, the position of scion variety or rootstock seems to affect the graft-take, in addition to other horticultural traits $[77,78]$. This reinforces the concept that grafting viability between genera within the Aurantioideae subfamily is difficult to predict and is very specific to the scion and rootstock genotypes and positions that are combined.

As expected, the highest rates of graft-take were observed for intrageneric combinations and most autografts, with the exception of Orange jasmine. Curry tree formerly belonged to the genus Murraya, and therefore, it is closely related to Orange jasmine. It showed better compatibility results for intergeneric and autograft combinations in comparison with Orange jasmine. Curry trees grafted on Rangpur lime and on Pomeroy and Swingle trifoliate genotypes had a good initial graft-take and survived up to 12 months, regardless of the position of the graft in the combination. However, they showed low vegetative growth and development, which declined later. Despite the relatively short survival period, these combinations can help to better understand the mechanisms involved in the incompatibility between Murraya (subtribe Clauseninae) and Citrus / Poncirus (subtribe Citrinae). In addition, it allows the evaluation of key aspects of CLas resistance and movement within plant tissues because the Curry tree is immune to the pathogen [8,34]. Therefore, it may be challenged as an ideal resistant rootstock or model plant [79].

Although inverted T-budding is the most popular method for citrus propagation [80], cleft grafting was chosen because it was an easier technique to implement when using young plants. This technique was also selected because several of the genera evaluated in this study had a stem bark that did not slip readily. Commercial citrus nursery trees show graft-take at rates higher than $95 \%$ and show great vigor when grown in the greenhouse [80]. During the current study, these graft-take rates were only attained by intrageneric citrus grafts and most autografts. The combinations Rangpur/Tabog, Wampee/Rangpur, Wampee/Pomeroy, Wampee/Swingle, Rangpur/Wampee, Pomeroy/Wampee, Swingle/ Wampee, and Tabog/Box orange presented with successful grafts and thus have potential for use in other studies and further field evaluations. The graft incompatibility of citrus 
varies considerably, not only due to the species/varieties used but also because of effects of the environmental and management conditions. The assessment of all these factors together may take more than four years to confirm the occurrence of successful grafting in the field [81,82]. This must take place prior to making any recommendations for commercial use. In addition, the unexpected putative graft compatibility of Swingle citrumelo with phylogenetically distant species must be highlighted in such a study. Despite being a rootstock of extensive commercial use [83], there are many reports of graft incompatibility of Swingle with different citrus genotypes used as scions [84].

The sweet orange varieties evaluated in the current study were graft-incompatible with most of the citrus relatives tested. However, interstocking with a third genotype that is compatible with both sweet orange and citrus relatives could be an alternative for overcoming this issue. In this context, trifoliate genotypes, in particular Swingle citrumelo, showed good compatibility with Clausena lansium. In consequence, it could be evaluated as a compatible interstock between sweet orange and Wampee. Although Wampee has been reported as a host of CLas, with very low bacterial titers [54,55], mother trees close to the experimental area did not show visual HLB symptoms. The bacterium was never detected by qPCR testing, even after 12 years in the field (results not shown; Figure S3). The use of C. lansium as a rootstock or interstock associated with Swingle citrumelo may be used as a model combination for CLas resistance/tolerance studies. Sweet orange varieties compatible with Swingle citrumelo should be grafted on top as the scion variety leading to viable horticultural combinations. Moreover, another species of the genus Clausena, such as C. excavata, which was described as immune to the CLas bacterium [8], may also be widely screened for graft compatibility with Citrus and related genera. Tabog is another species that shows some potential as a rootstock of citrus, which may allow for a low multiplication level of CLas in the root tissues [33]. Recently, accessions and hybrids of Microcitrus and Eremocitrus within Citrinae were demonstrated to be resistant to CLas, with the advantage of being both sexually and graft-compatible with Citrus [7]. Taken together, these new discoveries broaden the range of potential uses for CLas-resistant rootstocks of citrus. In addition, these could be used to facilitate the breeding of new resistant varieties with the aim of decreasing the impact of HLB on the industry. Moreover, these species will help in understanding important issues of CLas pathogenicity, such as the association between losses in root biomass and symptoms visible on the canopy of diseased plants [36].

The graft combinations with the highest rates of graft-take and vegetative growth in the greenhouse were transplanted into the field. Twelve months after planting, most plants showed suitable survival and growth rates. A complementary evaluation of CLas incidence due to natural infection showed that $50 \%$ of the plants of the Hamlin sweet orange/Rangpur lime combination were already infected, despite frequent spraying of insecticide to control the insect vector. No other combination tested positive for CLas at the time, confirming the higher susceptibility of Citrus to HLB [8,41]. Nevertheless, all CLas-resistant genotype combinations that were evaluated as scion or rootstocks for this experiment need continued monitoring for several seasons. This allows further evaluation of CLas incidence and plant responses to the pathogen, as well as validation of their horticultural performance.

In conclusion, biometric traits and multivariate clustering were successful in screening and classifying Aurantioideae species for graft compatibility. Anatomically, it became clear that incompatibility within this subfamily is characterized by a deficient differentiation of xylem-derived calli associated with a sharp buildup of phenolic compounds at the graft union. Although most intergeneric combinations were graft-incompatible, Rangpur/Tabog, Tabog/Rangpur, Wampee/Rangpur, Wampee/Pomeroy, Wampee/Swingle, Pomeroy/Wampee, and Swingle/Wampee were selected as being promising for further assessment under greenhouse and field conditions. Taken together, the results herein suggest that broadening the evaluation to other intrageneric and intergeneric combinations within Aurantioideae is worthwhile. Graft compatibility seems to be highly dependent on the genotypes combined, sometimes irrespective of their genetic contiguity. Since graft compatibility is of pivotal importance for citrus propagation, there is still a clear need for a 
greater understanding of this phenomenon within citrus and its relatives, aiming for better exploration of germplasm resources.

Supplementary Materials: The following supporting information can be downloaded at https: / / www.mdpi.com/article/10.3390/agriculture12010076/s1, Figure S1: Plants of (left) Citrus reticulata grafted on Murraya paniculata, and (right) M. paniculata grafted on C. reticulata two months after grafting. Figure S2: Plants of (left) Rangpur lime grafted on Clausena lansium, and (right) Clausena lansium grafted on Rangpur lime 12 months after transplanting into the field. Figure S3: Twelve years-old Clausena lansium mother trees did not show visual HLB symptoms in field cultivation close to the experimental area, and the 'Candidatus Liberibacter asiaticus' bacterium was never detected by qPCR.

Author Contributions: Conceptualization, L.L.R.-J., E.A.G. and L.P.; methodology, L.L.R.-J., J.P.R.M. and E.A.G.; software, A.S.M. and E.V.d.C.; validation, L.L.R.-J. and E.A.G.; formal analysis, L.L.R.-J., A.S.M., E.V.d.C. and J.P.R.M.; investigation, L.L.R.-J., E.V.d.C. and J.P.R.M.; resources, E.S.S., L.P. and E.A.G.; data curation, L.L.R.-J. and E.A.G.; writing-original draft preparation, L.L.R.-J., A.S.M., E.V.d.C. and J.P.R.M.; writing-review and editing, E.S.S., L.P. and E.A.G.; visualization, L.L.R.-J., J.P.R.M. and A.S.M.; supervision, E.A.G.; project administration, L.P.; funding acquisition, E.S.S., L.P. and E.A.G. All authors have read and agreed to the published version of the manuscript.

Funding: This research was funded by Fundecitrus (GA 303, 304, and 317. 041641), Embrapa (MP 22.16.04.030.00.00 and 30.21.90.043.00.00), and H2020 from the European Union (GA 817526).

Data Availability Statement: The dataset for this research is available upon reasonable request to the corresponding author.

Acknowledgments: The authors are grateful to Fundecitrus and Fundação Coopercitrus Credicitrus for technical support, plant materials and field, laboratory, and greenhouse infrastructure; to the Laboratory of Electron Microscopy "Elliot Watanabe Kitajima" for the infrastructure of the digital microscopy facility necessary for conducting the anatomical studies; and to Silvio Aparecido Lopes and Nelson Arno Wulff from Fundecitrus, for their suggestions and critical comments.

Conflicts of Interest: The authors declare no conflict of interest.

\section{References}

1. Swingle, W.T.; Reece, P.C. The botany of Citrus and its wild relatives. In The Citrus Industry, 2nd ed.; Reuther, W., Webber, H.J., Batchelor, D.L., Eds.; University of California Press: Berkeley, CA, USA, 1967; Volume 1, pp. 190-430.

2. $\quad$ Engler, A. Rutaceae. In Die Naturlichen Pflanzenfamilian, 2nd ed.; Engler, A., Prantl, K., Eds.; Engelmann: Leipzig, Germany, 1931; Volume 19a, pp. 187-359.

3. Tanaka, T. The taxonomy and nomenclature of Rutaceae Aurantioideae. Blumea 1936, 2, 101-110.

4. Wu, G.A.; Sugimoto, C.; Kinjo, H.; Azama, C.; Mitsube, F.; Talon, M.; Gmitter, F.G.; Rokhsar, D.S. Diversification of mandarin citrus by hybrid speciation and apomixis. Nat. Commun. 2021, 12, 4377. [CrossRef]

5. Wu, G.A.; Terol, J.; Ibanez, V.; López-García, A.; Estela, P.-R.; Carles, B.; Domingo, C.; Tadeo, F.R.; Carbonell-Caballero, J.; Alonso, R.; et al. Genomics of the origin and evolution of Citrus. Nature 2018, 554, 311-316. [CrossRef] [PubMed]

6. Spiegel-Roy, P.; Goldschmidt, E.E. Biology of Citrus; Cambridge University Press: Cambridge, UK, 1996 ; p. 244.

7. Alves, M.N.; Lopes, S.A.; Raiol-Junior, L.L.; Wulff, N.A.; Girardi, E.A.; Ollitrault, P.; Peña, L. Resistance to 'Candidatus Liberibacter asiaticus', the Huanglongbing Associated Bacterium, in Sexually and/or Graft-Compatible Citrus Relatives. Front. Plant Sci. 2021, 11, 617664. [CrossRef]

8. Ramadugu, C.; Keremane, M.L.; Halbert, S.E.; Duan, Y.P.; Roose, M.L.; Stover, E.; Lee, R.F. Long-Term Field Evaluation Reveals HLB Resistance in Citrus Relatives. Plant Dis. 2016, 100, 1858-1869. [CrossRef] [PubMed]

9. Swingle, W.T. The botany of Citrus and its wild relatives of the orange subfamily. In The Citrus Industry, 1st ed.; Batchelor, L.D., Webber, H.J., Eds.; University of California Press: Berkeley, CA, USA, 1943; Volume 1, pp. 128-474.

10. Krueger, R.R.; Navarro, L. Citrus germplasm resources. In Citrus Genetics, Breeding and Biotechnology, 1st ed.; Khan, I.A., Ed.; CABI: Wallington, NJ, UAS, 2007; pp. 45-140.

11. Barrett, H.C. Hybridization of citrus and related genera. Fruit Var. J. 1985, 39, 11-16.

12. Iwamasa, M.; Nito, N.; Ling, J.T. Intra- and intergeneric hybridization in the orange subfamily, Aurantioideae. In Proceedings of the Sixth Intetnet Citrus Congr, Middle-East, Tel Aviv, Israel, 6-11 March 1988; Goren, R., Mendel, K., Eds.; Margraf Scientific Books: Weikersheim, Germany, 1988; pp. 123-130.

13. Siebert, T.J.; Kahn, T.L.; Krueger, R.R. Observations of graft compatibility between Citrus spp. and related Aurantioideae taxa. Acta Hortic. 2015, 1065, 173-179. [CrossRef] 
14. Yoshida, T. Graft Compatibility of Citrus with Plants in the Aurantioideae and Their Susceptibility to Citrus Tristeza Virus. Plant Dis. 1996, 80, 414-417. [CrossRef]

15. Russo, F. Portinnesti e propagazione. In Trattato di Agrumicoltura; Cutuli, G., Di Martino, E., Lo Giudice, V., Pennisi, L., Raciti, G., Russo, F., Scuderi, A., Spina, P., Eds.; Edagricole: Bologna, Italy, 1985; pp. 289-322.

16. Bitters, W.P.; Cole, D.A.; Brusca, J.A. Citrus relatives are not irrelevant as dwarfing stocks or interstocks for citrus. Proc. Int. Soc. Citric. 1977, 2, 561-567.

17. Bitters, W.P.; Cole, D.A.; Brusca, J.A. The citrus relatives as citrus rootstocks. Proc. First Int. Citrus Symp. 1969, 1, $411-415$.

18. Bitters, W.P.; Brusca, J.A.; Cole, D.A. The search for new citrus rootstocks. Citrograph 1964, 49, 443-448.

19. Loupit, G.; Cookson, S.J. Identifying Molecular Markers of Successful Graft Union Formation and Compatibility. Front. Plant Sci. 2020, 11, 610352. [CrossRef]

20. Lima, L.K.S.; Soares, T.L.; de Souza, E.H.; de Jesus, O.N.; Girardi, E.A. Initial vegetative growth and graft region anatomy of yellow passion fruit on Passiflora spp. rootstocks. Sci. Hortic. 2017, 215, 134-141. [CrossRef]

21. Pina, A.; Zhebentyayeva, T.; Errea, P.; Abbott, A. Isolation and molecular characterization of cinnamate 4-hydroxylase from apricot and plum. Biol. Plant. 2012, 56, 441-450. [CrossRef]

22. Assunção, M.; Santos, C.; Brazão, J.; Eiras-Dias, J.E.; Fevereiro, P. Understanding the molecular mechanisms underlying graft success in grapevine. BMC Plant Biol. 2019, 19, 396. [CrossRef]

23. Chen, Z.; Zhao, J.; Hu, F.; Qin, Y.; Wang, X.; Hu, G. Transcriptome changes between compatible and incompatible graft combination of Litchi chinensis by digital gene expression profile. Sci. Rep. 2017, 7, 3954. [CrossRef] [PubMed]

24. Irisarri, P.; Zhebentyayeva, T.; Errea, P.; Pina, A. Differential expression of phenylalanine ammonia lyase (PAL) genes implies distinct roles in development of graft incompatibility symptoms in Prunus. Sci. Hortic. 2016, 204, 16-24. [CrossRef]

25. Pina, A.; Cookson, S.; Calatayud, A.; Trinchera, A.; Errea, P. Physiological and molecular mechanisms underlying graft compatibility. In Vegetable Grafting: Principles and Practices, 1st ed.; Colla, G., Perez-Alfocea, F., Schwarz, D., Eds.; CAB International: Cambridge, UK, 2017; pp. 132-154.

26. Prabpree, A.; Sangsil, P.; Nualsri, C.; Nakkanong, K. Expression profile of phenylalanine ammonia-lyase (PAL) and phenolic content during early stages of graft development in bud grafted Hevea brasiliensis. Biocatal. Agric. Biotechnol. 2018, 14, 88-95 [CrossRef]

27. Xu, Q.; Guo, S.-R.; Li, H.; Du, N.-S.; Shu, S.; Sun, J. Physiological Aspects of Compatibility and Incompatibility in Grafted Cucumber Seedlings. J. Am. Soc. Hortic. Sci. 2015, 140, 299-307. [CrossRef]

28. Wang, J.; Jin, Z.; Yin, H.; Yan, B.; Ren, Z.Z.; Xu, J.; Mu, C.J.; Zhang, Y.; Wang, M.Q.; Liu, H. Auxin redistribution and shifts in PIN gene expression during Arabidopsis grafting. Russ. J. Plant Physiol. 2014, 61, 688-696. [CrossRef]

29. Samuolienè, G.; Viškelienè, A.; Sirtautas, R.; Kviklys, D. Relationships between apple tree rootstock, crop-load, plant nutritional status and yield. Sci. Hortic. 2016, 211, 167-173. [CrossRef]

30. Clemente-Moreno, M.J.; Hevin, C.; Ollat, N.; Cookson, S.J. Developments at the graft interface in homo- and hetero-grafts. Plant Signal. Behav. 2014, 9, e28852. [CrossRef] [PubMed]

31. Goldschmidt, E.E. Plant grafting: New mechanisms, evolutionary implications. Front. Plant Sci. 2014, 5, 727. [CrossRef] [PubMed]

32. Mudge, K.; Janick, J.; Scofield, S.; Goldschmidt, E.E. A history of grafting. Hortic. Rev. 2009, 35, 437-493. [CrossRef]

33. Arenas, J.C.C.; Beattie, G.A.C.; Peña, L.; Lopes, S.A. Murraya paniculata and Swinglea glutinosa as Short-Term Transient Hosts of 'Candidatus Liberibacter asiaticus' and Implications for the Spread of Huanglongbing. Phytopathology 2019, 109, $2064-2073$. [CrossRef] [PubMed]

34. Beloti, V.H.; Alves, G.R.; Coletta-Filho, H.D.; Yamamoto, P.T. The Asian Citrus Psyllid Host Murraya koenigii Is Immune to Citrus Huanglongbing Pathogen 'Candidatus Liberibacter asiaticus'. Phytopathology 2018, 108, 1089-1094. [CrossRef] [PubMed]

35. Bové, J.M. Huanglongbing: A destructive, newly-emerging, century-old disease of citrus. J. Plant Pathol. 2006, 88, 7-37. [CrossRef]

36. Johnson, E.G.; Wu, J.; Bright, D.B.; Graham, J.H. Association of 'Candidatus Liberibacter asiaticus' root infection, but not phloem plugging with root loss on huanglongbing-affected trees prior to appearance of foliar symptoms. Plant Pathol. 2014, 63, 290-298. [CrossRef]

37. Albrecht, U.; Bowman, K.D. Reciprocal influences of rootstock and scion citrus cultivars challenged with Ca. Liberibacter asiaticus. Sci. Hortic. 2019, 254, 133-142. [CrossRef]

38. Albrecht, U.; Bowman, K.D. Tolerance of trifoliate citrus rootstock hybrids to Candidatus Liberibacter asiaticus. Sci. Hortic. 2012, 147, 71-80. [CrossRef]

39. Albrecht, U.; Mccollum, G.; Bowman, K.D. Influence of rootstock variety on Huanglongbing disease development in field-grown sweet orange (Citrus sinensis [L.] Osbeck) trees. Sci. Hortic. 2012, 138, 210-220. [CrossRef]

40. Albrecht, U.; Bowman, K.D. Tolerance of the Trifoliate Citrus Hybrid US-897 (Citrus reticulata Blanco $\times$ Poncirus trifoliata L. Raf.) to Huanglongbing. HortScience 2011, 46, 16-22. [CrossRef]

41. Folimonova, S.Y.; Robertson, C.J.; Garnsey, C.S.M.; Gowda, S.; Dawson, W.O. Examination of the Responses of Different Genotypes of Citrus to Huanglongbing (Citrus Greening) under Different Conditions. Phytopathology 2009, 99, 1346-1354. [CrossRef]

42. Yokomi, R.K.; Selvaraj, V.; Maheshwari, Y.; Saponari, M.; Giampetruzzi, A.; Chiumenti, M.; Hajeri, S. Identification and Characterization of Citrus tristeza virus Isolates Breaking Resistance in Trifoliate Orange in California. Phytopathology 2017, 107, 901-908. [CrossRef] [PubMed] 
43. Garnsey, S.M.; Barrett, H.C.; Hutchison, D.J. Identification of citrus tristeza virus resistance in citrus relatives and its potential applications. Phytophylactica 1987, 19, 187-191.

44. Lopes, S.A. Scion Substitution: A New Strategy to Control Citrus Variegated Chlorosis Disease. Plant Dis. 2020, 104, 239-245. [CrossRef] [PubMed]

45. Garcia, A.L.; Torres, S.C.Z.; Heredia, M.; Lopes, S.A. Citrus Responses to Xylella fastidiosa Infection. Plant Dis. 2012, 96, 1245-1249. [CrossRef]

46. Matheron, M.E.; Wright, G.C.; Porchas, M. Resistance to Phytophthora citrophthora and P. parasitica and Nursery Characteristics of Several Citrus Rootstocks. Plant Dis. 1998, 82, 1217-1225. [CrossRef] [PubMed]

47. Wutscher, H.K. Citrus rootstocks. Hortic. Rev. 1979, 1, 237-269.

48. Dalbó, M.A.; Arioli, C.J.; Lopes da Silva, M. Resistance of rootstocks to grapevine decline and dieback in southern Brazil. Acta Hortic. 2016, 1127, 65-70. [CrossRef]

49. Keller, M. The Science of Grapevines: Anatomy and Physiology, 3rd ed.; Academic Press: Cambridge, MA, USA, $2010 ;$ p. 400.

50. Louws, F.J.; Rivard, C.L.; Kubota, C. Grafting fruiting vegetables to manage soilborne pathogens, foliar pathogens, arthropods and weeds. Sci. Hortic. 2010, 127, 127-146. [CrossRef]

51. King, S.R.; Davis, A.R.; Liu, W.; Levi, A. Grafting for Disease Resistance. HortScience 2008, 43, 1673-1676. [CrossRef]

52. Norelli, J.L.; Holleran, H.T.; Johnson, W.C.; Robinson, T.L.; Aldwinckle, H.S. Resistance of Geneva and Other Apple Rootstocks to Erwinia amylovora. Plant Dis. 2003, 87, 26-32. [CrossRef] [PubMed]

53. Ollitrault, P.; Curk, F.; Krueger, R. Citrus Taxonomy. In The Genus Citrus, 1st ed.; Talon, M., Caruso, M., Gmitter, F.G., Eds.; Elsevier: Duxford, UK, 2020; pp. 57-81. [CrossRef]

54. Deng, X.; Zhou, G.; Li, H.; Chen, J.; Civerolo, E.L. Detection of Candidatus Liberibacter asiaticus from Wampee (Clausena lansium Skeels) by Nested PCR. Plant Health Prog. 2007, 8, 53. [CrossRef]

55. Ding, F.; Wang, G.; Yi, G.; Zhong, Y.; Zeng, J.; Zhou, B. Infection of wampee and lemon by the citrus huanglongbing pathogen (Candidatus Liberibacter asiaticus) in China. J. Plant Pathol. 2005, 87, 207-212. [CrossRef]

56. Hung, T.-H.; Wu, M.-L.; Su, H.-J. Identification of the Chinese Box Orange (Severinia Buxifolia) as an Alternative Host of the Bacterium Causing Citrus Huanglongbing. Eur. J. Plant Pathol. 2001, 107, 183-189. [CrossRef]

57. Webber, H. Rootstocks: Their characters and reactions. In The Citrus Industry; Webber, H.J., Batchelor, L.D., Eds.; University of California Press: Berkeley, CA, USA, 1948; Volume 2, pp. 70-80.

58. Marques, J.P.R.; Hoy, J.W.; Appezzato-Da-Glória, B.; Viveros, A.F.G.; Vieira, M.L.C.; Baisakh, N. Sugarcane Cell Wall-Associated Defense Responses to Infection by Sporisorium scitamineum. Front. Plant Sci. 2018, 9, 698. [CrossRef] [PubMed]

59. Rolim, G.D.S.; de Camargo, M.B.P.; Lania, D.G.; de Moraes, J.F.L. Classificação climática de Köppen e de Thornthwaite e sua aplicabilidade na determinação de zonas agroclimáticas para o estado de são Paulo. Bragantia 2007, 66, 711-720. [CrossRef]

60. Murray, M.G.; Thompson, W.F. Rapid isolation of high molecular weight plant DNA. Nucleic Acids Res. 1980, 8, 4321-4325. [CrossRef] [PubMed]

61. Teixeira, D.D.C.; Danet, J.L.; Eveillard, S.; Martins, E.C.; Junior, W.C.D.J.; Yamamoto, P.T.; Lopes, S.A.; Bassanezi, R.B.; Ayres, A.J.; Saillard, C.; et al. Citrus huanglongbing in São Paulo State, Brazil: PCR detection of the 'Candidatus' Liberibacter species associated with the disease. Mol. Cell. Probes 2005, 19, 173-179. [CrossRef] [PubMed]

62. Zheng, Z.; Xu, M.; Bao, M.; Wu, F.; Chen, J.; Deng, X. Unusual Five Copies and Dual Forms of nrdB in "Candidatus Liberibacter asiaticus": Biological Implications and PCR Detection Application. Sci. Rep. 2016, 6, 39020. [CrossRef] [PubMed]

63. R Core Team. A Language and Environment for Statistical Computing; R Foundation for Statistical Computing: Vienna, Austria, 2015; Available online: http://www.R-project.org/ (accessed on 1 October 2021).

64. Conrado, T.V.; Ferreira, D.F.; Scapim, C.A.; Maluf, W.R.; Ufla, B. Adjusting the Scott-Knott cluster analyses for unbalanced designs. Crop. Breed. Appl. Biotechnol. 2017, 17, 1-9. [CrossRef]

65. Scott, R.J.; Knott, M. A Cluster Analysis Method for Grouping Means in the Analysis of Variance. Biometrics 1974, 30, 507-512. [CrossRef]

66. Hair, J.F.; Black, W.C.; Babin, B.J.; Anderson, R.E. Multivariate Data Analysis, 7th ed.; Pearson Prentice Hall: Upper Saddle River, NJ, USA, 2009; p. 761.

67. Charrad, M.; Ghazzali, N.; Boiteau, V.; Niknafs, A.; Charrad, M.M. NbClust: An R package for determining the relevant number of clusters in a data set. J. Stat. Softw. 2014, 61, 1-36. [CrossRef]

68. Lê, S.; Josse, J.; Husson, F. FactoMineR: An R Package for Multivariate Analysis. J. Stat. Softw. 2008, 25, 1-18. [CrossRef]

69. Kaiser, H.F. The varimax criterion for analytic rotation in factor analysis. Psychometrika 1958, 23, 187-200. [CrossRef]

70. Calvez, L.; Dereeper, A.; Mournet, P.; Froelicher, Y.; Bruyère, S.; Morillon, R.; Ollitrault, P. Intermediate Inheritance with Disomic Tendency in Tetraploid Intergeneric Citrus $x$ Poncirus Hybrids Enhances the Efficiency of Citrus Rootstock Breeding. Agronomy 2020, 10, 1961. [CrossRef]

71. Grosser, J.W.; Gmitter, F.G. Protoplast fusion in the production of tetraploids and triploids: Applications in scion and rootstock breeding. Plant Cell Tissue Organ Cult. 2011, 104, 343-357. [CrossRef]

72. Ćalović, M.; Chen, C.; Yu, Q.; Orbović, V.; Gmitter, F.G.; Grosser, J.W. New Somatic Hybrid Mandarin Tetraploids Generated by Optimized Protoplast Fusion and Confirmed by Molecular Marker Analysis and Flow Cytometry. J. Am. Soc. Hortic. Sci. 2019, 144, 151-163. [CrossRef] 
73. Usenik, V.; Krška, B.; Vičan, M.; Štampar, F. Early detection of graft incompatibility in apricot (Prunus armeniaca L.) using phenol analyses. Sci. Hortic. 2006, 109, 332-338. [CrossRef]

74. Santoro, M.B.; Brogio, B.D.A.; Bueno, S.C.S.; Tanaka, F.A.O.; Jacomino, A.P.; da Silva, S.R. Vegetative propagation of Campomanesia phaea by the air-layering and grafting techniques. Pesqui. Agropecuária Bras. 2021, 56, e02402. [CrossRef]

75. Vahdati, K.; Sarikhani, S.; Arab, M.M.; Leslie, C.A.; Dandekar, A.M.; Aletà, N.; Bielsa, B.; Gradziel, T.M.; Montesinos, Á.; Rubio-Cabetas, M.J.; et al. Advances in Rootstock Breeding of Nut Trees: Objectives and Strategies. Plants 2021, $10,2234$. [CrossRef]

76. Pérez-Luna, A.; Prieto-Ruíz, J.Á.; López-Upton, J.; Carrillo-Parra, A.; Wehenkel, C.; Chávez-Simental, J.A.; Hernández-Díaz, J.C. Some Factors Involved in the Success of Side Veneer Grafting of Pinus engelmannii Carr. Forests 2019, 10, 112. [CrossRef]

77. Santana-Vieira, D.; Freschi, L.; Almeida, L.; Moraes, D.H.S.; Neves, D.M.; Santos, L.M.; Bertolde, F.Z.; Soares Filho, W.S.; Coelho Filho, M.A.; Gesteira, A.S. Survival strategies of citrus rootstocks subjected to drought. Sci. Rep. 2016, 6, 38775. [CrossRef]

78. Huang, Y.; Bie, Z.L.; Liu, P.Y.; Niu, M.L.; Zhen, A.; Liu, Z.X.; Lei, B.; Gu, D.J.; Lu, C.; Wang, B.T. Reciprocal grafting between cucumber and pumpkin demonstrates the roles of the rootstock in the determination of cucumber salt tolerance and sodium accumulation. Sci. Hortic. 2013, 149, 47-54. [CrossRef]

79. Raiol-Junior, L.L.; Cifuentes-Arenas, J.C.; Carvalho, M.E.V.; Girardi, E.A.; Lopes, S.A. Evidence That ‘Candidatus Liberibacter asiaticus' Moves Predominantly Toward New Tissue Growth in Citrus Plants. Plant Dis. 2021, 105, 34-42. [CrossRef] [PubMed]

80. De Carvalho, S.A.; Girardi, E.A.; Mourão Filho, F.D.A.A.; Ferrarezi, R.S.; Coletta Filho, H.D. Advances in citrus propagation in Brazil. Rev. Bras. Frutic. 2019, 41, e422. [CrossRef]

81. Pompeu Junior, J.; Blumer, S. Híbridos de trifoliata como porta-enxertos para laranjeira Pêra. Pesqui. Agropecu. Trop. 2014, 44, 9-14. [CrossRef]

82. Pompeu Junior, J. Porta-enxertos. In Citros, 1st ed.; Mattos Junior, D., De Negri, J.D., Pio, R.M., Pompeu Junior, J., Eds.; Instituto Agronômico (Fundag): Campinas, Brazil, 2005; pp. 61-104.

83. Girardi, E.A.; Pompeu Junior, J.; Sobrinho, J.T.; Soares Filho, W.S.; Passos, O.S.; Cristofani-Yaly, M.; Sampionato, O.R.; Stuchi, E.S.; Donadio, L.C.; Mattos Junior, D.; et al. Guia de Reconhecimento dos Citros em Campo: Um Guia Prático Para o Reconhecimento em Campo de Variedades de Laranjeira-Doce e Outras Espécies de Citros Cultivadas No Estado de São Paulo e Triângulo Mineiro, 1st ed.; Fundecitrus: Araraquara, Brazil, 2021; p. 158.

84. Garnsey, S.M.; Castle, W.S.; Tucker, D.P. Budunion incompatibilities and associated declines observed in Florida among trees on Swingle citrumelo and other trifoliate orange-related rootstocks. Proc. Fla. State Hort. Soc. 2001, 114, $121-127$. 